\title{
Random Attractors for Stochastic Reaction-Diffusion Equations with Distribution Derivatives on Unbounded Domains
}

\author{
Eshag Mohamed Ahmed, Ali Dafallah Abdelmajid, Ling Xu, Qiaozhen Ma* \\ College of Mathematics and Statistics, Northwest Normal University, Lanzhou, China \\ Email: ahmedesag@gmail.com, majid dafallah@yahoo.com, 13893414055@163.com, ํmaqzh@nwnu.edu.cn
}

Received 10 August 2015; accepted 22 September 2015; published 25 September 2015

Copyright (C) 2015 by authors and Scientific Research Publishing Inc.

This work is licensed under the Creative Commons Attribution International License (CC BY).

http://creativecommons.org/licenses/by/4.0/

(c) (i) Open Access

\begin{abstract}
In this paper, we prove the existence of random attractors for a stochastic reaction-diffusion equation with distribution derivatives on unbounded domains. The nonlinearity is dissipative for large values of the state and the stochastic nature of the equation appears spatially distributed temporal white noise. The stochastic reaction-diffusion equation is recast as a continuous random dynamical system and asymptotic compactness for this demonstrated by using uniform estimates far-field values of solutions. The results are new and appear to be optimal.
\end{abstract}

\section{Keywords}

Stochastic Reaction-Diffusion Equation, Random Attractors, Distribution Derivatives, Asymptotic Compactness, Unbounded Domain

\section{Introduction}

The understanding of the asymptotic behavior of dynamical system is one of the most important problems of modern mathematical physics; one way to attack the problem for dissipative deterministic dynamical systems is to consider its global attractors. This is an invariant set that attracts all the trajectories of the system. Its geometry can be very complicated and reflects the complexity of the long-time dynamical of the systems. In this paper we investigate the asymptotic behavior of solutions to the following stochastic reaction-diffusion equations with distribution derivatives and additive noise defined in the space $\mathbb{R}^{n}$ :

${ }^{*}$ Corresponding author.

How to cite this paper: Ahmed, E.M., Abdelmajid, A.D., Xu, L. and Ma, Q.Z. (2015) Random Attractors for Stochastic Reaction-Diffusion Equations with Distribution Derivatives on Unbounded Domains. Applied Mathematics, 6, $1790-1807$.

http://dx.doi.org/10.4236/am.2015.610159 


$$
\mathrm{d} u+(\lambda u-\Delta u) \mathrm{d} t=\left(f(u)+g(x)+D_{j} g^{j}\right) \mathrm{d} t+\sum_{j=1}^{m} h_{j} \mathrm{~d} w_{j},
$$

with initial data

$$
u(0, x)=u_{0}(x) \text { in } \mathbb{R}^{n},
$$

where $\lambda$ is a positive constant; $D_{j}=\frac{\partial}{\partial x_{j}}$ is distribution derivatives; $g^{j}, g \in L^{2}\left(\mathbb{R}^{n}\right)(j=1, \cdots, n) ; f$ is a nonlinear function satisfying certain dissipative conditions; $h_{j}$ is given functions defined on $\mathbb{R}^{n}$; and $\left\{w_{j}\right\}_{j=1}^{m}$ is independent two sided real-valued wiener processes on probability space which will be specified later.

Stochastic differential equations of this type arise from many physical systems when random spatio-temporal forcing is taken into account. In order to capture the essential dynamics of random systems with wide fluctuations, the concept of pullback random attractors was introduced in [1], being an extension to stochastic systems of the theory of attractors for deterministic equations found in [2]-[5], for instance. The existence of such random attractors has been studied for stochastic PDE on bounded domains; see, e.g. [6] [7], and for stochastic PDE on unbounded domains, see, e.g. [8] [9], and the references therein. In the present paper, we prove the existence of such a random attractor for stochastic reaction-diffusion Equation (1.1) defined in $\mathbb{R}^{n}$ which is not founded.

Notice that the unboundedness of domain introduces a major difficulty for proving the existence of an attractor because Sobolev embedding theorem is no longer compact and so the asymptotic compactness of solutions cannot be obtained by the standard method. In the case of deterministic equations, this difficulty can be overcome by the energy equation approach, introduced by Ball in [10] and then employed by several authors to prove the asymptotic compactness of deterministic equations in unbounded domains. This idea was developed in [5] to prove asymptotic compactness for the deterministic version of (1.1) on $\mathbb{R}^{n}$. In this paper, we provide uniform estimates on the far-field values of solutions to circumvent the difficulty caused by the unboundedness of the domains. The main contribution of this paper is to extend the method of using tail estimates of the case stochastic dissipative PDEs and prove the existence of random attractor for the stochastic reaction-diffusion equation with distribution derivatives on the unbounded domain $\mathbb{R}^{n}$.

The paper is organized as follows. In Section 2, we recall some preliminaries and abstract results on the existence of a pullback random attractor for random dynamical systems. In Section 3, we transform (1.1) into a continuous random dynamical system. Section 4 is devoted to obtain the uniform estimates of solution as $t \rightarrow \infty$. These estimates are necessary for proving the existence of bounded absorbing sets and the asymptotic compactness of the equation. In Section 5, we first establish the asymptotic compactness of the solution operator by giving uniform estimates on the tails of solutions, and then prove the estimates of a random attractor.

We denote by $\|\cdot\|$ and $(\cdot, \cdot)$ the norm and the inner product in $L^{2}\left(\mathbb{R}^{n}\right)$ and use $\|\cdot\|_{p}$ to denote the norm in $L^{p}\left(\mathbb{R}^{n}\right)$. Otherwise, the norm of a general Banach space $X$ is written as $\|\cdot\|_{X}$. The letters $C$ and $C_{i}(i=1,2, \cdots)$ are generic positive constants which may change their values form line to line or even in the same line.

\section{Preliminaries and Abstract Results}

As mentioned in the introduction, our main purpose is to prove the existence of a random attractor. For that matter, first, we will recapitulate basic concepts related to random attractors for stochastic dynamical systems. The reader is referred to [6] [11]-[13] for more details. Let $\left(X,\|\cdot\|_{X}\right)$ be a separable Hilbert space with Borel $\sigma$-algebra $B(X)$, and let $(\Omega, F, P)$ be a probability space.

Definition 2.1. $\left(\Omega, F, P,\left(\theta_{t}\right)_{t \in \mathbb{R}}\right)$ is called a metric dynamical system if $\theta: \mathbb{R} \times \Omega \rightarrow \Omega$ is $(B(\mathbb{R}) \times F, F)$ measurable, $\theta_{0}$ is the identity on $\Omega, \theta_{s+t}=\theta_{t} \circ \theta_{s}$ for all $s, t \in \mathbb{R}$ and $\theta_{0} P=P$ for all $t \in \mathbb{R}$.

Definition 2.2. A continuous random dynamical system (RDS) on $X$ over a metric dynamical system $\left(\Omega, F, P,\left(\theta_{t}\right)_{t \in \mathbb{R}}\right)$ is a mapping

$$
\phi: \mathbb{R}^{+} \times \Omega \times X \rightarrow X,(t, \omega, x) \rightarrow \phi(t, \omega, x),
$$

which is $\left(B\left(\mathbb{R}^{+}\right) \times F \times B(X), B(X)\right)$-measurable and satisfies, for P-a.e. $\omega \in \Omega$, (1) $\phi(0, \omega, \cdot)$ is the identity on $X ;$; (2) $\phi(t+s, \omega, \cdot)=\phi\left(t, \theta_{s} \omega, \cdot\right) \circ \phi(s, \omega, \cdot)$ for all $t, s \in \mathbb{R}^{+}$(3) $\phi(t, \omega, \cdot): X \rightarrow X$ is continuous for all $t \in \mathbb{R}^{+}$. Hereafter, we always assume that $\phi$ is a continuous RDS on $X$ over $\left(\Omega, F, P,\left(\theta_{t}\right)_{t \in \mathbb{R}}\right)$. 
Definition 2.3. A random bounded set $\{B(\omega)\}_{\omega \in \Omega}$ of $X$ is called tempered with respect to $\left(\theta_{t}\right)_{t \in \mathbb{R}}$ if for P-a.e $\omega \in \Omega$,

$$
\lim _{t \rightarrow \infty} \mathrm{e}^{-\beta t} d\left(B\left(\theta_{-t} \omega\right)\right)=0 \text { for all } \beta>0
$$

where $d(B)=\sup _{x \in B}\|x\|_{X}$.

Definition 2.4. Let $D$ be a collection of random subsets of $X$ and $\{K(\omega)\}_{\omega \in \Omega} \in D$. Then $\{K(\omega)\}_{\omega \in \Omega}$ is called a random absorbing set for $\phi$ in $D$ if for every $B \in D$ and P-a.e $\omega \in \Omega$, there exists $t_{B}(\omega)>0$ such that

$$
\phi\left(t, \theta_{-t} \omega, B\left(\theta_{-t} \omega\right)\right) \subseteq K(\omega) \text { for all } t \geq t_{B}(\omega) .
$$

Definition 2.5. Let $D$ be a collection of random subsets of $X$. Then $\phi$ is said to be $D$-pullback asymptotically compact in $X$ if for P-a.e $\omega \in \Omega,\left\{\phi\left(t_{n}, \theta_{-t_{n}} \omega, x_{n}\right)\right\}_{n=1}^{\infty}$ has a convergent subsequence in $X$ whenever $t_{n} \rightarrow \infty$, and $x_{n} \in B\left(\theta_{-t_{n}} \omega\right)$ with $\{B(\omega)\}_{\omega \in \Omega} \in D$.

Definition 2.6. Let $D$ be a collection of random sunsets of $X$. Then a random set $\{A(\omega)\}_{\omega \in \Omega}$ of $X$ is called a $D$-random attractor (or $D$-pullback attractor) for $\phi$ if the following conditions are satisfied, for P-a.e. $\omega \in \Omega$, (1) $A(\omega)$ is compact, and $\omega \mapsto d(x, A(\omega))$ is measurable for every $x \in X$; (2) $\{A(\omega)\}_{\omega \in \Omega}$ is invariant, that is,

$$
\phi(t, \omega, A(\omega))=A\left(\theta_{t} \omega\right) \text { for all } t \geq 0 \text {; }
$$

(3) $\{A(\omega)\}_{\omega \in \Omega}$ attracts every set in $D$, that is, for every $B=\{B(\omega)\}_{\omega \in \Omega} \in D$,

$$
\lim _{t \rightarrow \infty} d\left(\phi\left(t, \theta_{-t} \omega, B\left(\theta_{-t} \omega\right)\right), A(\omega)\right)=0,
$$

where $d$ is the Hausdorff semi-metric given by $d(Y, Z)=\sup _{y \in Y} \inf _{z \in Z}\|y-z\|_{X}$ for any $Y \subseteq X$ and $Z \subseteq X$. The following existence result for a random attractor for a continuous RDS can be found in [8] [13]. First, recall that a collection $D$ of random subsets is called inclusion closed if whenever $E(\omega)_{\omega \in \Omega}$ is an arbitrary random set, and $F(\omega)_{\omega \in \Omega}$ is in $D$ with $E(\omega) \subset F(\omega)$ for all $\omega \in \Omega$, then $E(\omega)_{\omega \in \Omega}$ must belong to $D$.

Definition 2.7. Let $D$ be an inclusion-closed collection of random subsets of $X$ and $\phi$ a continuous RDS on $X$ over $\left(\Omega, F, P,\left(\theta_{t}\right)_{t \in \mathbb{R}}\right)$. Suppose that $\{K(\omega)\}_{\omega \in \Omega}$ is a closed random absorbing set for $\phi$ in $D$ and $\phi$ is $D$-pullback asymptotically compact in $X$. Then $\phi$ has a unique $D$-random attractor $\{A(\omega)\}_{\omega \in \Omega}$ which is given by

$$
A(\omega)=\bigcap_{t \geq 0 s \geq t} \overline{\phi\left(t, \theta_{-t} \omega, K\left(\theta_{-t} \omega\right)\right)} .
$$

In this paper, we will take $D$ as the collection of all tempered random subsets of $L^{2}\left(\mathbb{R}^{n}\right)$ and prove the stochastic reaction-diffusion equation in $\mathbb{R}^{n}$ has a $D$-random attractor.

\section{The Reaction-Diffusion Equation on $\mathbb{R}^{n}$ with Distribution Derivatives and Additive Noise}

$$
\mathrm{d} u+(\lambda u-\Delta u) \mathrm{d} t=\left(f(u)+g(x)+D_{j} g^{j}\right) \mathrm{d} t+\sum_{j=1}^{m} h_{j} \mathrm{~d} w_{j}, x \in \mathbb{R}^{n}, t>0
$$

with initial condition

$$
u(x, 0)=u_{0}(x) \quad x \in \mathbb{R}^{n}
$$

where $\lambda$ is a positive constant, $g^{j}, g \in L^{2}\left(\mathbb{R}^{n}\right), h_{j} \in H^{2}\left(\mathbb{R}^{n}\right) \cap W^{2, p}\left(\mathbb{R}^{n}\right)$, for some $p \geq 2, D_{j}=\frac{\partial}{\partial x_{j}}$ are distribution derivative, $\left\{w_{j}\right\}_{j=1}^{m}$ are independent two-side real-valued wiener processes on a probability space which will be specified below, and $f \in C^{1}(\mathbb{R})$ with the following assumptions: 


$$
\begin{aligned}
& -\alpha_{2}|s|^{p}-k_{2}|s|^{2} \leq f(s) s \leq-\alpha_{1}|s|^{p}+k_{1}|s|^{2}, \\
& -\alpha_{4}|s|^{p-1}-k_{4}|s| \leq f(s) \leq-\alpha_{3}|s|^{p-1}+k_{3}|s|, \\
& \left|f^{\prime}(s)\right| \leq L,
\end{aligned}
$$

for $s \in \mathbb{R}$ and $p \geq 2$, where $L, \alpha_{1}, \cdots, \alpha_{4}, k_{1}, \cdots, k_{4}$ are positive constants and $\lambda>2 k_{1}+k_{4}$.

In the sequel, we consider the probability space $(\Omega, F, P)$ where

$$
\Omega=\left\{\omega=\left(\omega_{1}, \omega_{2}, \cdots, \omega_{m}\right) \in C\left(\mathbb{R}, \mathbb{R}^{m}\right): \omega(0)=0\right\} .
$$

$F$ is the Borel $\sigma$-algebra induced by the compact-open topology of $\Omega$, and $P$ the corresponding wiener measure on $(\Omega, F)$. Then we identify $\omega$ with

$$
W(t) \equiv\left(\omega_{1}(t), \omega_{2}(t), \cdots, \omega_{m}(t)\right)=\omega(t) \text { for } t \in \mathbb{R} .
$$

Define the time shift by

$$
\theta_{t} \omega(\cdot)=\omega(\cdot+t)-\omega(t), \quad \omega \in \Omega, \quad t \in \mathbb{R} .
$$

Then $\left(\Omega, F, P,\left(\theta_{t}\right)_{t \in \mathbb{R}}\right)$ is a metric dynamical system.

We now associate a continuous random dynamical system with the stochastic reaction-diffusion equation over $\left(\Omega, F, P,\left(\theta_{t}\right)_{t \in \mathbb{R}}\right)$. To this end, we need to convert the stochastic equation with a random additive term in to a deterministic equation with a random parameter. Given $j=1, \cdots, m$ consider the One-dimensional Ornsteinuhlenbeck equation

$$
\mathrm{d} z_{j}+\lambda z_{j} \mathrm{~d} t=\mathrm{d} w_{j}(t)
$$

The solution of (3.6) is given by

$$
z_{j}=z_{j}\left(\theta_{t} \omega_{j}\right) \equiv-\lambda \int_{-\infty}^{0} \mathrm{e}^{\lambda \tau\left(\theta_{t} \omega_{j}\right)}(\tau) \mathrm{d} \tau, t \in \mathbb{R} .
$$

Note that the random variable $\left|z_{j}\left(\omega_{j}\right)\right|$ is tempered and $z_{j}\left(\theta_{t} \omega_{j}\right)$ is P-a.e continuous, therefore, it follows form proposition 4.3.3 in [11] that there exists a tempered function $r(\omega)>0$ such that

$$
\sum_{j=1}^{m}\left(\left|z_{j}\left(\omega_{j}\right)\right|^{2}+\left|z_{j}\left(\omega_{j}\right)\right|^{p}\right) \leq r(\omega),
$$

where $r(\omega)$ satisfies for P-a.e $\omega \in \Omega$

$$
r\left(\theta_{t} \omega\right) \leq \mathrm{e}^{\frac{\lambda}{2}|t|} r(\omega), t \in \mathbb{R} .
$$

Then it follows form (3.7), (3.8) that, for P-a.e. $\omega \in \Omega$

$$
\sum_{j=1}^{m}\left(\left|z_{j}\left(\theta_{t} \omega_{j}\right)\right|^{2}+\left|z_{j}\left(\theta_{t} \omega_{j}\right)\right|^{p}\right) \leq \mathrm{e}^{\frac{\lambda}{2}|t|} r(\omega), t \in \mathbb{R} .
$$

Putting $z\left(\theta_{t} \omega\right)=\sum_{j=1}^{m} h_{j} z_{j}\left(\theta_{t} \omega_{j}\right)$, by (3.6) we have

$$
\mathrm{d} z+\lambda z \mathrm{~d} t=\sum_{j=1}^{m} h_{j} \mathrm{~d} w_{j} .
$$

The existence and uniqueness of solutions to the stochastic partial differential Equation (3.1) with initial condition (3.2) which can be obtained by standard Fatou-Galerkin methods. To show that problem (3.1), (3.2) generates a random system, we let $v(t)=u(t)-z\left(\theta_{t} \omega\right)$ where $u$ is a solution of problem (3.1), (3.2), then $v$ satisfies

$$
\frac{\partial v}{\partial t}+\lambda v-\Delta v=f\left(v+z\left(\theta_{t} \omega\right)\right)+g+D_{j} g^{j}+\Delta z\left(\theta_{t} \omega\right) .
$$

By a Galerkin method, one can show that if $f$ satisfies (3.3)-(3.5), then in the case of a bounded domain with Dirichlet boundary conditions, for P-a.e. $\omega \in \Omega$, and for all $v_{0} \in L^{2}$, (3.10) has a unique solution 


$$
v\left(\cdot, \omega, v_{0}\right) \in C\left([0, \infty), L^{2}\right) \cap L^{2}\left((0, T) ; H^{1}\right)
$$

with $v\left(0, \omega, v_{0}\right)=v_{0}$ for every $T>0$, one may take the domain to be a sequence of Balls with radius approaching $\infty$ to deduce the existence of a weak solution to (3.10) on $\mathbb{R}^{n}$, further, one may show that $v\left(t, \omega, v_{0}\right)$ is unique and continuous with respect to $v_{0}$ in $L^{2}\left(\mathbb{R}^{n}\right)$ for all $t \geq 0$. Let

$$
u\left(t, \omega, u_{0}\right)=v\left(t, \omega, u_{0}-z(\omega)\right)+z\left(\theta_{t} \omega\right) .
$$

Then the process $u$ is the solution of problem (3.1), (3.2), we now define a mapping $\phi: \mathbb{R}^{+} \times \Omega \times L^{2}\left(\mathbb{R}^{n}\right) \rightarrow L^{2}\left(\mathbb{R}^{n}\right)$ by

$$
\phi\left(t, \omega, u_{0}\right)=u\left(t, \omega, u_{0}\right)=v\left(t, \omega, u_{0}-z(\omega)\right)+z\left(\theta_{t} \omega\right) \text { for all }\left(t, \omega, u_{0}\right) \in \mathbb{R}^{+} \times \Omega \times L^{2}\left(\mathbb{R}^{n}\right) .
$$

Then $\phi$ is satisfies conditions (1)-(3) in Definition 2.2 therefore $\phi$ is a continuous random dynamical system associated with the stochastic reaction-diffusion equation on $\mathbb{R}^{n}$. In the next two sections, we establish uniform estimates for the solutions of problem (3.1), (3.2) and prove the existence of a random attractor for $\phi$.

\section{Uniform Estimates of Solutions}

In this section, we drive uniform estimates on the solutions of (3.1), (3.2) defined on $\mathbb{R}^{n}$ when $t \rightarrow \infty$ with the purpose of proving the existence of a bounded random absorbing set and the asymptotic compactness of the random dynamical system associated with the equation. In particular, we will show that the tails of the solutions, i.e. solutions evaluated at large values of $|x|$, are uniformly small when the time is sufficiently large.

We always assume that $D$ is the collection of all tempered subsets of $L^{2}\left(\mathbb{R}^{n}\right)$ with respect to $\left(\Omega, F, P,\left(\theta_{t}\right)_{t \in \mathbb{R}}\right)$ the next lemma shows that $\phi$ has a random absorbing set in $D$.

Lemma 4.1. Assume that $g^{j}, g \in L^{2}\left(\mathbb{R}^{n}\right)$, and (3.3)-(3.5) hold. Then there exists $\{K(\omega)\}_{\omega \in \Omega} \in D$ such that $\{K(\omega)\}_{\omega \in \Omega}$ is a random absorbing set for $\phi$ in $D$, that is, for any $B=\{B(\omega)\}_{\omega \in \Omega} \in D$ and P-a.e $\omega \in \Omega$, there is $T_{B}(\omega)>0$ such that

$$
\phi\left(t, \theta_{-t} \omega, B\left(\theta_{-t} \omega\right)\right) \subseteq K(\omega) \text { for all } t \geq T_{B}(\omega) .
$$

Proof. We first derive uniform estimates on $v(t)=u(t)-z\left(\theta_{t} \omega\right)$ from which the uniform estimates on $u(t)$. Multipling (3.10) by $v$ and then integrating over $\mathbb{R}^{n}$, we have

$$
\frac{1}{2} \frac{\mathrm{d}}{\mathrm{d} t}\|v\|^{2}+\lambda\|v\|^{2}+\|\nabla v\|^{2}=\int_{\mathbb{R}^{n}} f\left(v+z\left(\theta_{t} \omega\right)\right) v \mathrm{~d} x+(g, v)+\left(\Delta z\left(\theta_{t} \omega\right), v\right)+\left(D_{j} g^{j}, v\right) .
$$

For the nonlinear term, by (3.3)-(3.5) we obtain

$$
\begin{aligned}
& \int_{\mathbb{R}^{n}} f\left(v+z\left(\theta_{t} \omega\right)\right) v \mathrm{~d} x \\
& =\int_{\mathbb{R}^{n}} f\left(v+z\left(\theta_{t} \omega\right)\right)\left(v+z\left(\theta_{t} \omega\right)\right) \mathrm{d} x-\int_{\mathbb{R}^{n}} f\left(v+z\left(\theta_{t} \omega\right)\right) z\left(\theta_{t} \omega\right) \mathrm{d} x \\
& =\int_{\mathbb{R}^{n}} f(u) u \mathrm{~d} x-\int_{\mathbb{R}^{n}} f(u) z\left(\theta_{t} \omega\right) \mathrm{d} x \\
& \leq-\alpha_{1} \int_{\mathbb{R}^{n}}|u|^{p} \mathrm{~d} x+k_{1} \int_{\mathbb{R}^{n}}|u|^{2} \mathrm{~d} x+\alpha_{4} \int_{\mathbb{R}^{n}}|u|^{p-1} z\left(\theta_{t} \omega\right) \mathrm{d} x+k_{4} \int_{\mathbb{R}^{n}}|u| z\left(\theta_{t} \omega\right) \mathrm{d} x \\
& \leq-\alpha_{1}\|u\|_{p}^{p}+k_{1}\|u\|^{2}+\alpha_{4} \int_{\mathbb{R}^{n}}\left(|u|^{p}\right)^{\frac{p-1}{p}} \cdot\left(\left|z\left(\theta_{t} \omega\right)\right|^{p}\right)^{\frac{1}{p}} \mathrm{~d} x+\frac{1}{2} k_{4}\|u\|^{2}+\frac{1}{2} k_{4}\left\|z\left(\theta_{t} \omega\right)\right\|^{2} \\
& \leq-\alpha_{1}\|u\|_{p}^{p}+k_{1}\|u\|^{2}+\frac{1}{2} \alpha_{1}\|u\|_{p}^{p}+C_{1}\left\|z\left(\theta_{t} \omega\right)\right\|_{p}^{p}+\frac{1}{2} k_{4}\|u\|^{2}+\frac{1}{2} k_{4}\left\|z\left(\theta_{t} \omega\right)\right\|^{2} \\
& \leq-\alpha_{1}\|u\|_{p}^{p}+\left(k_{1}+\frac{1}{2} k_{4}\right)\left(\|v\|^{2}+\left\|z\left(\theta_{t} \omega\right)\right\|^{2}\right)+\frac{1}{2} \alpha_{1}\|u\|_{p}^{p}+C_{1}\left\|z\left(\theta_{t} \omega\right)\right\|_{p}^{p}+\frac{1}{2} k_{4}\left\|z\left(\theta_{t} \omega\right)\right\|^{2} \\
& \leq-\frac{1}{2} \alpha_{1}\|u\|_{p}^{p}+\left(k_{1}+\frac{1}{2} k_{4}\right)\|v\|^{2}+C_{2}\left(\left\|z\left(\theta_{t} \omega\right)\right\|_{p}^{p}+\left\|z\left(\theta_{t} \omega\right)\right\|^{2}\right),
\end{aligned}
$$

on the other hand, the next two terms on the right-hand side of (4.1) are bounded by 


$$
\|g\|\|v\|+\left\|\nabla z\left(\theta_{t} \omega\right)\right\|\|\nabla v\| \leq \frac{1}{2} \lambda\|v\|^{2}+\frac{1}{2 \lambda}\|g\|^{2}+\frac{1}{2}\left\|\nabla z\left(\theta_{t} \omega\right)\right\|^{2}+\frac{1}{2}\|\nabla v\|^{2},
$$

the last term on the right-hand side of (4.1) is bounded by

$$
\left|\left\langle D_{j} g^{j}, v\right\rangle\right| \leq|g||\nabla v| \leq \frac{1}{2}\|\tilde{g}\|^{2}+\frac{1}{2}\|\nabla v\|^{2}
$$

where $\tilde{g}=\left(g^{1}, \cdots, g^{n}\right)$ and $\|\tilde{g}\|^{2}=\sum_{j=1}^{n}\left|g^{j}\right|^{2}$.
Then it follows from (4.1)-(4.4) that

$$
\begin{aligned}
& \frac{\mathrm{d}}{\mathrm{d} t}\|v\|^{2}+\lambda\|v\|^{2}+\alpha_{1}\|u\|_{p}^{p}-\left(2 k_{1}+k_{4}\right)\|v\|^{2} \\
& \leq \frac{1}{\lambda}\|g\|^{2}+\|\tilde{g}\|^{2}+\left\|\nabla z\left(\theta_{t} \omega\right)\right\|^{2}+2 C_{2}\left(\left\|z\left(\theta_{t} \omega\right)\right\|_{p}^{p}+\left\|z\left(\theta_{t} \omega\right)\right\|^{2}\right) .
\end{aligned}
$$

Note that $z\left(\theta_{t} \omega\right)=\sum_{j=1}^{m} h_{j} z_{j}\left(\theta_{t} \omega_{j}\right)$ and $h_{j} \in H^{2}\left(\mathbb{R}^{n}\right) \cap W^{2, p}\left(\mathbb{R}^{n}\right)$, therefore, the right-hand side of (4.5) is bounded as following

$$
C_{3} \sum_{j=1}^{m}\left(\left\|z_{j}\left(\theta_{t} \omega_{j}\right)\right\|^{p}+\left\|z_{j}\left(\theta_{t} \omega_{j}\right)\right\|^{2}\right)+C_{4}=P_{1}\left(\theta_{t} \omega\right)+C_{4} .
$$

By (3.9), we find that for P-a.e, $\omega \in \Omega$

$$
P_{1}\left(\theta_{\tau} \omega\right) \leq C_{3} \mathrm{e}^{\frac{1}{2} \lambda|\tau|} r(\omega) \text { for all } \tau \in \mathbb{R}
$$

it follows from (4.5), (4.6) that, all $t \geq 0$,

$$
\frac{\mathrm{d}}{\mathrm{d} t}\|v\|^{2}+\lambda\|v\|^{2}+\alpha_{1}\|u\|_{p}^{p}-\left(2 k_{1}+k_{4}\right)\|v\|^{2} \leq P_{1}\left(\theta_{t} \omega\right)+C_{4}
$$

which implies that for all $t \geq 0$,

$$
\frac{\mathrm{d}}{\mathrm{d} t}\|v\|^{2}+\left(\lambda-2 k_{1}-k_{4}\right)\|v\|^{2} \leq P_{1}\left(\theta_{t} \omega\right)+C_{4} .
$$

Let $\lambda_{1}=\lambda-2 k_{1}-k_{4}$. Applying Gronwall's lemma, we find that, for all $t \geq 0$,

$$
\left\|v\left(t, \omega, v_{0}(\omega)\right)\right\|^{2} \leq \mathrm{e}^{-\lambda_{1} t}\left\|v_{0}(\omega)\right\|^{2}+\int_{0}^{t} \mathrm{e}^{\lambda_{1}(\tau-t)} P_{1}\left(\theta_{\tau} \omega\right) \mathrm{d} \tau+\frac{C_{4}}{\lambda_{1}} .
$$

By replacing $\omega$ by $\theta_{-t} \omega$, we get from (4.10) and (4.7) that for all $t \geq 0$

$$
\left\|v\left(t, \theta_{-t} \omega\right), v_{0}\left(\theta_{-t} \omega\right)\right\|^{2} \leq \mathrm{e}^{-\lambda_{1} t}\left\|v_{0}\left(\theta_{-t} \omega\right)\right\|^{2}+\frac{2 C_{3}}{\lambda_{1}} r(\omega)+\frac{C_{4}}{\lambda_{1}} .
$$

Note that $\phi\left(t, \omega, u_{0}(\omega)\right)=v\left(t, \omega, u_{0}(\omega)-z(\omega)\right)+z\left(\theta_{t} \omega\right)$.

So from (4.11) we get that, for all $t \geq 0$,

$$
\begin{aligned}
& \left\|\phi\left(t, \theta_{-t} \omega, u_{0}\left(\theta_{-t} \omega\right)\right)\right\|^{2} \\
& =\left\|v\left(t, \theta_{-t} \omega, u_{0}\left(\theta_{-t} \omega\right)-z\left(\theta_{t} \omega\right)\right)+z(\omega)\right\|^{2} \\
& \leq 2\left\|v\left(t, \theta_{-t} \omega, u_{0}\left(\theta_{-t} \omega\right)-z\left(\theta_{-t} \omega\right)\right)\right\|^{2}+2\|z(\omega)\|^{2} \\
& \leq 2 \mathrm{e}^{-\lambda_{1} t}\left\|u_{0}\left(\theta_{-t} \omega\right)-z\left(\theta_{-t} \omega\right)\right\|^{2}+C_{3} r(\omega)+C_{4}+2\|z(\omega)\|^{2} \\
& \leq 4 \mathrm{e}^{-\lambda_{1} t}\left(\left\|u_{0}\left(\theta_{-t} \omega\right)\right\|^{2}+\left\|z\left(\theta_{-t} \omega\right)\right\|^{2}\right)+C_{3} r(\omega)+C_{4}+2\|z(\omega)\|^{2} .
\end{aligned}
$$

By assumption $\{B(\omega)\}_{\omega \in \Omega} \in D$ is tempered. On the other hand, by definition, $\|z(\omega)\|^{2}$ is also tempered, therefore, if $u_{0}\left(\theta_{-t} \omega\right) \in B\left(\theta_{-t} \omega\right)$. Then there is $T_{B}(\omega)>0$ such that for all $t \geq T_{B}(\omega)$ 


$$
4 \mathrm{e}^{-\lambda_{1} t}\left(\left\|u_{0}\left(\theta_{-t} \omega\right)\right\|^{2}+\left\|z\left(\theta_{-t} \omega\right)\right\|^{2}\right) \leq C_{3} r(\omega)+C_{4},
$$

which along with (4.12) shows that, for all $t \geq T_{B}(\omega)$,

$$
\left\|\phi\left(t, \theta_{-t} \omega, u_{0}\left(\theta_{-t} \omega\right)\right)\right\|^{2} \leq 2\left(C_{3} r(\omega)+C_{4}+\|z(\omega)\|^{2}\right) .
$$

Given $\omega \in \Omega$

$$
K(\omega)=\left\{u \in L^{2}\left(\mathbb{R}^{n}\right):\|u\|^{2} \leq 2\left(C_{3} r(\omega)+C_{4}+\|z(\omega)\|^{2}\right)\right\} .
$$

Then $\{K(\omega)\}_{\omega \in \Omega} \in D$, further, (4.13) indicates that $\{K(\omega)\}_{\omega \in \Omega}$ is a random absorbing set for $\phi$ in $D$. Which completes the Proof.

We next drive uniform estimates for $u$ in $H^{1}\left(\mathbb{R}^{n}\right)$ and for $u$ in $L^{p}\left(\mathbb{R}^{n}\right)$.

Lemma 4.2. Assume that $g \in L^{2}\left(\mathbb{R}^{n}\right)$ and (3.3)-(3.5) hold, let $B=\{B(\omega)\}_{\omega \in \Omega} \in D$ and $u_{0}(\omega) \in B(\omega)$. Then for every $T_{1} \geq 0$ and P-a.e $\omega \in \Omega$, the solutions $u\left(t, \omega, u_{0}(\omega)\right)$ of problem (3.1), (3.2) and $v\left(t, \omega, v_{0}(\omega)\right)$ of (3.11) with $v_{0}(\omega)=u_{0}(\omega)-z(\omega)$ satisfy, for all $t \geq T_{1}$.

$$
\begin{aligned}
& \int_{T_{1}}^{t} \mathrm{e}^{\lambda_{1}(s-t)}\left\|u\left(s, \theta_{-t} \omega, u_{0}\left(\theta_{-t} \omega\right)\right)\right\|_{p}^{p} \mathrm{~d} s \leq \mathrm{e}^{-\lambda_{1} t}\left\|\nu_{0}\left(\theta_{-t} \omega\right)\right\|^{2}+C(1+r(\omega)), \\
& \int_{T_{1}}^{t} \mathrm{e}^{\lambda_{1}(s-t)}\left\|u\left(s, \theta_{-t} \omega, u_{0}\left(\theta_{-t} \omega\right)\right)\right\|^{2} \mathrm{~d} s \leq \mathrm{e}^{-\lambda_{1} t}\left\|\nu_{0}\left(\theta_{-t} \omega\right)\right\|^{2}+C(1+r(\omega)),
\end{aligned}
$$

where $\mathrm{C}$ is a positive deterministic constant independent of $T_{1}$ and $r(\omega)$ is the tempered function in (3.7).

Proof. First, replacing $t$ by $T_{1}$ and then replacing $\omega$ by $\theta_{-t} \omega$ in (4.10) we find that

$$
\left\|v\left(T_{1}, \theta_{-t} \omega, v_{0}\left(\theta_{-t} \omega\right)\right)\right\|^{2} \leq \mathrm{e}^{-\lambda_{1} T_{1}}\left\|v_{0}\left(\theta_{-t} \omega\right)\right\|^{2}+\int_{0}^{T_{1}} \mathrm{e}^{\lambda_{1}\left(s-T_{1}\right)} P_{1}\left(\theta_{s-t} \omega\right) \mathrm{d} s+C .
$$

Multiply the above by $\mathrm{e}^{\lambda_{1}\left(T_{1}-t\right)}$ and then simplify to get.

$$
\begin{aligned}
& \mathrm{e}^{\lambda_{1}\left(T_{1}-t\right)}\left\|v\left(T_{1}, \theta_{-t} \omega, v_{0}\left(\theta_{-t} \omega\right)\right)\right\|^{2} \\
& \leq \mathrm{e}^{-\lambda_{1} t}\left\|v_{0}\left(\theta_{-t} \omega\right)\right\|^{2}+\int_{0}^{T_{1}} \mathrm{e}^{\lambda_{1}(s-t)} P_{1}\left(\theta_{s-t} \omega\right) \mathrm{d} s+C \mathrm{e}^{\lambda_{1}\left(T_{1}-t\right)} .
\end{aligned}
$$

By (4.7), the second term on the right-hand side of (4.16) satisfies

$$
\begin{aligned}
& \int_{0}^{T_{1}} \mathrm{e}^{\lambda_{1}(s-t)} P_{1}\left(\theta_{s-t} \omega\right) \mathrm{d} s \\
& =\int_{-t}^{T_{1}-t} \mathrm{e}^{\lambda_{1} \tau} P_{1}\left(\theta_{\tau} \omega\right) \mathrm{d} \tau \leq C_{3} r(\omega) \int_{-t}^{T_{1}-t} \mathrm{e}^{\frac{1}{2} \lambda_{1} \tau} \mathrm{d} \tau \leq \frac{2}{\lambda_{1}} C_{3} r(\omega) \mathrm{e}^{\frac{1}{2} \lambda_{1}\left(T_{1}-t\right)} .
\end{aligned}
$$

From (4.16), (4.17) it follows that

$$
\begin{aligned}
& \mathrm{e}^{\lambda_{1}\left(T_{1}-t\right)}\left\|v\left(T_{1}, \theta_{-t} \omega, v_{0}\left(\theta_{-t} \omega\right)\right)\right\|^{2} \\
& \leq \mathrm{e}^{-\lambda_{1} t}\left\|v_{0}\left(\theta_{-t} \omega\right)\right\|^{2}+\frac{2}{\lambda_{1}} C_{3} r(\omega) \mathrm{e}^{\frac{1}{2} \lambda_{1}\left(T_{1}-t\right)}+C \mathrm{e}^{\lambda_{1}\left(T_{1}-t\right)} .
\end{aligned}
$$

By (4.8) we find that, for $t \geq T_{1}$

$$
\begin{aligned}
& \left\|v\left(t, \omega, v_{0}(\omega)\right)\right\|^{2}+\alpha_{1} \int_{T_{1}}^{t} \mathrm{e}^{\lambda_{1}(s-t)}\left\|u\left(s, \omega, u_{0}(\omega)\right)\right\|_{p}^{p} \mathrm{~d} s \\
& +2 k_{1} \int_{T_{1}}^{t} \mathrm{e}^{\lambda_{1}(s-t)}\left\|u\left(s, \omega, u_{0}(\omega)\right)\right\|^{2} \mathrm{~d} s+k_{4} \int_{T_{1}}^{t} \mathrm{e}^{\lambda_{1}(s-t)}\left\|u\left(s, \omega, u_{0}(\omega)\right)\right\|^{2} \mathrm{~d} s \\
& \leq \mathrm{e}^{\lambda_{1}\left(T_{1}-t\right)}\left\|v\left(T_{1}, \omega, v_{0}(\omega)\right)\right\|^{2}+\int_{T_{1}}^{t} \mathrm{e}^{\lambda_{1}(s-t)} P_{1}\left(\theta_{s} \omega\right) \mathrm{d} s+C \int_{T_{1}}^{t} \mathrm{e}^{\lambda_{1}(s-t)} \mathrm{d} s .
\end{aligned}
$$

Dropping the first term on the left-hand side of (4.19) and replacing $\omega$ by $\theta_{-t} \omega$, we obtain that, for all $t \geq T_{1}$ 


$$
\begin{aligned}
& \alpha_{1} \int_{T_{1}}^{t} \mathrm{e}^{\lambda_{1}(s-t)}\left\|u\left(s, \theta_{-t} \omega, u_{0}\left(\theta_{-t} \omega\right)\right)\right\|_{p}^{p} \mathrm{~d} s+2 k_{1} \int_{T_{1}}^{t} \mathrm{e}^{\lambda_{1}(s-t)}\left\|u\left(s, \theta_{-t} \omega, u_{0}\left(\theta_{-t} \omega\right)\right)\right\|^{2} \mathrm{~d} s \\
& +k_{4} \int_{T_{1}}^{t} \mathrm{e}^{\lambda_{1}(s-t)}\left\|u\left(s, \theta_{-t} \omega, u_{0}\left(\theta_{-t} \omega\right)\right)\right\|^{2} \mathrm{~d} s \\
& \leq \mathrm{e}^{\lambda_{1}\left(T_{1}-t\right)}\left\|v\left(T_{1}, \theta_{-t} \omega, v_{0}\left(\theta_{-t} \omega\right)\right)\right\|^{2}+\int_{T_{1}-t}^{0} \mathrm{e}^{\lambda_{1} \tau} P_{1}\left(\theta_{\tau} \omega\right) \mathrm{d} \tau+\frac{C}{\lambda_{1}} .
\end{aligned}
$$

By (4.7), the second term on the right-hand side of (4.20) satisfies, for all $t \geq T_{1}$

$$
\int_{T_{1}-t}^{0} \mathrm{e}^{\lambda_{1} \tau} P_{1}\left(\theta_{\tau} \omega\right) \mathrm{d} \tau \leq C_{3} r(\omega) \int_{T_{1}-t}^{0} \mathrm{e}^{\frac{1}{2} \lambda_{1} \tau} \mathrm{d} \tau \leq \frac{2}{\lambda_{1}} C_{3} r(\omega)
$$

Then, using (4.20) and (4.21), it follows from (4.20) that

$$
\begin{aligned}
& \alpha_{1} \int_{T_{1}}^{t} \mathrm{e}^{\lambda_{1}(s-t)}\left\|u\left(s, \theta_{-t} \omega, u_{0}\left(\theta_{-t} \omega\right)\right)\right\|_{p}^{p} \mathrm{~d} s+2 k_{1} \int_{T_{1}}^{t} \mathrm{e}^{\lambda_{1}(s-t)}\left\|u\left(s, \theta_{-t} \omega, u_{0}\left(\theta_{-t} \omega\right)\right)\right\|^{2} \mathrm{~d} s \\
& +k_{4} \int_{T_{1}}^{t} \mathrm{e}^{\lambda_{1}(s-t)}\left\|u\left(s, \theta_{-t} \omega, u_{0}\left(\theta_{-t} \omega\right)\right)\right\|^{2} \mathrm{~d} s \leq \mathrm{e}^{-\lambda_{1} t}\left\|v_{0}\left(\theta_{-t} \omega\right)\right\|^{2}+C(1+r(\omega)) .
\end{aligned}
$$

This completes the proof.

Lemma 4.3. Assume that $g^{j}, g \in L^{2}\left(\mathbb{R}^{n}\right)$ and (3.3)-(3.5) hold, Let $B=\{B(\omega)\}_{\omega \in \Omega} \in D$ and $u_{0}(\omega) \in B(\omega)$. Then for P-a.e $\omega \in \Omega$, there exists $T_{B}(\omega)>0$ such that the solutions $u\left(t, \omega, u_{0}(\omega)\right)$ of problem (3.1), (3.2) and $v\left(t, \omega, v_{0}(\omega)\right)$ of (3.11) with $v_{0}(\omega)=u_{0}(\omega)-z(\omega)$ satisfy, for all $t \geq T_{B} \omega$.

$$
\begin{gathered}
\int_{t}^{t+1}\left\|u\left(s, \theta_{-t-1} \omega, u_{0}\left(\theta_{-t-1} \omega\right)\right)\right\|_{p}^{p} \mathrm{~d} s \leq C(1+r(\omega)), \\
\int_{t}^{t+1}\left\|u\left(s, \theta_{-t-1} \omega, u_{0}\left(\theta_{-t-1} \omega\right)\right)\right\|^{2} \mathrm{~d} s \leq C(1+r(\omega)),
\end{gathered}
$$

where $C$ is a positive deterministic constant and $r(\omega)$ is the tempered function in (3.7).

Proof. First replacing $t$ by $t+1$ and then replacing $T_{1}$ by $t$ in (4.14), we find that

$$
\int_{t}^{t+1} \mathrm{e}^{\lambda_{1}(s-t-1)}\left\|u\left(s, \theta_{-t-1} \omega, u_{0}\left(\theta_{-t-1} \omega\right)\right)\right\|_{p}^{p} \mathrm{~d} s \leq \mathrm{e}^{-\lambda_{1}(t+1)}\left\|\nu_{0}\left(\theta_{-t-1} \omega\right)\right\|^{2}+C(1+r(\omega)) .
$$

Note that $\mathrm{e}^{\lambda_{1}(s-t-1)} \geq \mathrm{e}^{-\lambda_{1}}$ for $s \in[t, t+1]$, hence, form (4.22) we have

$$
\begin{aligned}
& \mathrm{e}^{-\lambda_{1}} \int_{t}^{t+1}\left\|u\left(s, \theta_{-t-1} \omega, u_{0}\left(\theta_{-t-1} \omega\right)\right)\right\|_{p}^{p} \mathrm{~d} s \\
& \leq \mathrm{e}^{-\lambda_{1}(t+1)}\left\|v_{0}\left(\theta_{-t-1} \omega\right)\right\|^{2}+C(1+r(\omega)) \\
& \leq 2 \mathrm{e}^{-\lambda_{1}(t+1)}\left\|u_{0}\left(\theta_{-t-1} \omega\right)\right\|^{2}+\left\|z\left(\theta_{-t-1} \omega\right)\right\|^{2}+C(1+r(\omega)) .
\end{aligned}
$$

Since $\left\|u_{0}(\omega)\right\|^{2}$ and $\|z(\omega)\|^{2}$ are tempered there is $T_{B}(\omega)>0$ such that for all $t \geq T_{B}(\omega)$

$$
2 \mathrm{e}^{-\lambda_{1}(t+1)}\left(\left\|u_{0}\left(\theta_{-t-1} \omega\right)\right\|^{2}+\left\|z\left(\theta_{-t-1} \omega\right)\right\|^{2}\right) \leq C(1+r(\omega)),
$$

which along with (4.23) shows that, for all $t \geq T_{B}(\omega)$,

$$
\int_{t}^{t+1}\left\|u\left(s, \theta_{-t-1} \omega, u_{0}\left(\theta_{-t-1} \omega\right)\right)\right\|_{p}^{p} \mathrm{~d} s \leq 2 \mathrm{e}^{\lambda_{1}} C(1+r(\omega)) .
$$

Then from (4.10) using the same steps of last process applying on (4.15), we get that

$$
\int_{t}^{t+1}\left\|u\left(s, \theta_{-t-1} \omega, u_{0}\left(\theta_{-t-1} \omega\right)\right)\right\|^{2} \mathrm{~d} s \leq 2 \mathrm{e}^{\lambda_{1}} C(1+r(\omega)) .
$$

The above uniform estimates is a special case lemma 4.2, then the lemma follows from (4.24)-(4.25). $\square$.

Lemma 4.4. Assume that $g^{j}, g \in L^{2}\left(\mathbb{R}^{n}\right)$ and (3.3)-(3.5) hold, let $B=\{B(\omega)\}_{\omega \in \Omega} \in D$ and $u_{0}(\omega) \in B(\omega)$. Then for P-a.e $\omega \in \Omega$, there exists $T_{B}(\omega)>0$ such that the solution $u\left(t, \omega, u_{0}(\omega)\right)$ of problem (3.1), (3.2) satisfies, for all $t \geq T_{B}(\omega)$. 


$$
\int_{t}^{t+1}\left\|\nabla u\left(s, \theta_{-t-1} \omega, u_{0}\left(\theta_{-t-1} \omega\right)\right)\right\|^{2} \mathrm{~d} s \leq C(1+r(\omega)),
$$

where $C$ is a positive deterministic constant and $r(\omega)$ is the tempered function in (3.9).

Proof. Let $T_{B}(\omega)$ be the positive constant in lemma 4.3, take $t \geq T_{B}(\omega)$ and $s \in(t, t+1)$, by (3.11) we find that

$$
\begin{aligned}
& \left\|\nabla u\left(s, \theta_{-t-1} \omega, u_{0}\left(\theta_{-t-1} \omega\right)\right)\right\|^{2} \\
& =\left\|\nabla v\left(s, \theta_{-t-1} \omega, u_{0}\left(\theta_{-t-1} \omega\right)\right)+\nabla z\left(\theta_{s-t-1} \omega\right)\right\|^{2} \\
& \leq 2\left\|\nabla v\left(s, \theta_{-t-1} \omega, u_{0}\left(\theta_{-t-1} \omega\right)\right)\right\|^{2}+2\left\|\nabla z\left(\theta_{s-t-1} \omega\right)\right\|^{2} .
\end{aligned}
$$

By (3.9) we obtain

$$
2\left\|\nabla z\left(\theta_{s-t-1} \omega\right)\right\|^{2} \leq C \sum_{j=1}^{m}\left|z_{j}\left(\theta_{s-t-1} \omega_{j}\right)\right|^{2} \leq C \mathrm{e}^{\frac{1}{2}(t+1-s)} r(\omega) \leq C \mathrm{e}^{\frac{\lambda_{1}}{2} r(\omega)} .
$$

Now integration (4.26) with respect to s over $(t, t+1)$, by lemma 4.3 and inequality (4.27), we have

$$
\int_{t}^{t+1}\left\|\nabla u\left(s, \theta_{-t-1} \omega, u_{0}\left(\theta_{-t-1} \omega\right)\right)\right\|^{2} \mathrm{~d} s \leq C_{5}+C_{6} r(\omega)
$$

Then the lemma follows from (4.28).

Lemma 4.5. Assume that $g^{j}, \quad g \in L^{2}\left(\mathbb{R}^{n}\right)$ and (3.3)-(3.5) hold, let $B=\{B(\omega)\}_{\omega \in \Omega} \in D$ and $u_{0}(\omega) \in B(\omega)$. Then for P-a.e $\omega \in \Omega$, there exists $T_{B}(\omega)>0$ such that for all $t \geq T_{B} \omega$.

$$
\left\|\nabla u\left(t, \theta_{-t-1} \omega, u_{0}\left(\theta_{-t-1} \omega\right)\right)\right\|^{2} \leq C(1+r(\omega))
$$

where $C$ is a positive deterministic constant and $r(\omega)$ is the tempered function in (3.9).

Proof. Taking the inner product of (3.10) with $\Delta v$ in $L^{2}\left(\mathbb{R}^{n}\right)$, we get that

$$
\begin{aligned}
& \frac{1}{2} \frac{\mathrm{d}}{\mathrm{d} t}\|\nabla v\|^{2}+\lambda\|\nabla v\|^{2}+\|\Delta v\|^{2} \\
& =\int_{\mathbb{R}^{n}} f(u) \Delta v \mathrm{~d} x+\left(g+\Delta z\left(\theta_{t} \omega\right), \Delta v\right)+\left(D_{j} g^{j}, \Delta v\right) .
\end{aligned}
$$

We estimates the first term in the right-hand side of (4.29) by (3.3), (3.4) we have

$$
\begin{aligned}
\int_{\mathbb{R}^{n}} f(u) \Delta v \mathrm{~d} x & =\int_{\mathbb{R}^{n}} f(u) \Delta u-\int_{\mathbb{R}^{n}} f(u) \Delta z\left(\theta_{t} \omega\right) \mathrm{d} x \\
& =-\int_{\mathbb{R}^{n}} \frac{\partial f}{\partial u}(u)|\nabla u|^{2} \mathrm{~d} x-\int_{\mathbb{R}^{n}} f(u) \Delta z\left(\theta_{t} \omega\right) \mathrm{d} x \\
& \leq L\|\nabla u\|^{2}+\alpha_{4} \int_{\mathbb{R}^{n}}|u|^{p-1}\left|\Delta z\left(\theta_{t} \omega\right)\right| \mathrm{d} x+k_{4} \int_{\mathbb{R}^{n}}\left|u \| \Delta z\left(\theta_{t} \omega\right)\right| \mathrm{d} x \\
& \leq L\|\nabla u\|^{2}+\frac{\alpha_{4}(p-1)}{p} \int_{\mathbb{R}^{n}}|u|^{p} \mathrm{~d} x+\frac{\alpha_{4}}{p} \int_{\mathbb{R}^{n}}\left|\Delta z\left(\theta_{t} \omega\right)\right|^{p} \mathrm{~d} x+k_{4}\|u\|^{2}+k_{4}\left\|\Delta z\left(\theta_{t} \omega\right)\right\|^{2} \\
& \leq L\|\nabla u\|^{2}+\frac{\alpha_{4}(p-1)}{p}\|u\|_{p}^{p}+\frac{\alpha_{4}}{p}\left\|\Delta z\left(\theta_{t} \omega\right)\right\|_{p}^{p}+k_{4}\|u\|^{2}+k_{4}\left\|\Delta z\left(\theta_{t} \omega\right)\right\|^{2} \\
& \leq C\left(\|u\|^{2}+\|\nabla u\|^{2}+\|u\|_{p}^{p}\right)+C\left(\left\|\Delta z\left(\theta_{t} \omega\right)\right\|_{p}^{p}+\left\|\Delta z\left(\theta_{t} \omega\right)\right\|^{2}\right) .
\end{aligned}
$$

On the other hand, the second term on the right-hand side of (4.29) is bounded by

$$
|(g, \Delta v)|+\left|\left(\Delta z\left(\theta_{t} \omega\right), \Delta v\right)\right| \leq \frac{1}{2}\|\Delta v\|^{2}+\|g\|^{2}+\left\|\Delta z\left(\theta_{t} \omega\right)\right\|^{2} .
$$

The last term on the right-hand side of (4.29) is bounded by

$$
\left|\left(D_{j} g^{j}, \Delta v\right)\right| \leq\|\nabla \tilde{g}\|\|\Delta v\| \leq \frac{1}{2}\|\nabla \tilde{g}\|^{2}+\frac{1}{2}\|\Delta v\|^{2} .
$$


By (4.29)-(4.32) we get that

$$
\begin{aligned}
& \frac{\mathrm{d}}{\mathrm{d} t}\|\nabla v\|^{2}+2 \lambda\|\nabla v\|^{2} \\
& \leq C\left(\|u\|^{2}+\|\nabla u\|^{2}+\|u\|_{p}^{p}\right)+C\left(\left\|\Delta z\left(\theta_{t} \omega\right)\right\|_{p}^{p}+\left\|\Delta z\left(\theta_{t} \omega\right)\right\|^{2}\right) \\
& \quad+2\|g\|^{2}+2\|\nabla \tilde{g}\|^{2}+2\left\|\Delta z\left(\theta_{t} \omega\right)\right\|^{2} \\
& \leq C\left(\|u\|^{2}+\|\nabla u\|^{2}+\|u\|_{p}^{p}\right)+C\left(\left\|\Delta z\left(\theta_{t} \omega\right)\right\|_{p}^{p}+\left\|\Delta z\left(\theta_{t} \omega\right)\right\|^{2}+1\right) .
\end{aligned}
$$

Let

$$
P_{2}\left(\theta_{t} \omega\right)=C\left(\left\|\Delta z\left(\theta_{t} \omega\right)\right\|_{p}^{p}+\left\|\Delta z\left(\theta_{t} \omega\right)\right\|^{2}+1\right) .
$$

Since $z\left(\theta_{t} \omega\right)=\sum_{j=1}^{m} h_{j} z_{j}\left(\theta_{t} \omega_{j}\right)$ and $h_{j} \in H^{2}\left(\mathbb{R}^{n}\right) \cap W^{2, p}\left(\mathbb{R}^{n}\right)$, there are positive constants $C_{1}$ and $C_{2}$ such that

$$
P_{2}\left(\theta_{t} \omega\right) \leq C_{1} \sum_{j=1}^{m}\left(\left|z_{j}\left(\theta_{t} \omega_{j}\right)\right|_{p}^{p}+\left|z_{j}\left(\theta_{t} \omega_{j}\right)\right|^{2}\right)+C_{2},
$$

which along with (3.9) shows that

$$
P_{2}\left(\theta_{t} \omega\right) \leq C_{1} \mathrm{e}^{\frac{1}{2}|t|} r(\omega)+C_{2} \text { for all } t \in \mathbb{R}
$$

By (4.33), (4.34) we have

$$
\frac{\mathrm{d}}{\mathrm{d} t}\|\nabla v\|^{2} \leq C\left(\|u\|^{2}+\|\nabla u\|^{2}+\|u\|_{p}^{p}\right)+P_{2}\left(\theta_{t} \omega\right) .
$$

Let $T_{B}(\omega)$ be the positive constant in lemma 4.3 take $t \geq T_{B}(\omega)$ and $s \in(t, t+1)$. Then integrate 4.36 over $(s, t+1)$ to get that

$$
\begin{aligned}
& \left\|\nabla v\left(t+1, \omega, v_{0}(\omega)\right)\right\|^{2} \leq\left\|\nabla v\left(s, \omega, v_{0}(\omega)\right)\right\|^{2}+\int_{s}^{t+1} p_{2}\left(\theta_{\tau} \omega\right) \mathrm{d} \tau \\
& +C \int_{s}^{t+1}\left(\left\|u\left(\tau, \omega, u_{0}(\omega)\right)\right\|^{2}+\left\|\nabla u\left(\tau, \omega, u_{0}(\omega)\right)\right\|^{2}+\left\|u\left(\tau, \omega, u_{0}(\omega)\right)\right\|_{p}^{p}\right) \mathrm{d} \tau \\
& \leq\left\|\nabla v\left(s, \omega, v_{0}(\omega)\right)\right\|^{2}+\int_{t}^{t+1} p_{2}\left(\theta_{\tau} \omega\right) \mathrm{d} \tau \\
& +C \int_{t}^{t+1}\left(\left\|u\left(\tau, \omega, u_{0}(\omega)\right)\right\|^{2}+\left\|\nabla u\left(\tau, \omega, u_{0}(\omega)\right)\right\|^{2}+\left\|u\left(\tau, \omega, u_{0}(\omega)\right)\right\|_{p}^{p}\right) \mathrm{d} \tau .
\end{aligned}
$$

Now integrating the above equation with respect to s over $(t, t+1)$, we find that

$$
\begin{aligned}
& \left\|\nabla v\left(t+1, \omega, v_{0}(\omega)\right)\right\|^{2} \leq \int_{t}^{t+1}\left\|\nabla v\left(s, \omega, v_{0}(\omega)\right)\right\|^{2} \mathrm{~d} s+\int_{t}^{t+1} p_{2}\left(\theta_{\tau} \omega\right) \mathrm{d} \tau \\
& +C \int_{t}^{t+1}\left(\left\|u\left(\tau, \omega, u_{0}(\omega)\right)\right\|^{2}+\left\|\nabla u\left(\tau, \omega, u_{0}(\omega)\right)\right\|^{2}+\left\|u\left(\tau, \omega, u_{0}(\omega)\right)\right\|_{p}^{p}\right) \mathrm{d} \tau .
\end{aligned}
$$

Replacing $\omega$ by $\theta_{-t-1} \omega$ we obtain that

$$
\begin{aligned}
& \left\|\nabla v\left(t+1, \theta_{-t-1} \omega, v_{0}\left(\theta_{-t-1} \omega\right)\right)\right\|^{2} \\
& \leq \int_{t}^{t+1}\left\|\nabla v\left(s, \theta_{-t-1} \omega, v_{0}\left(\theta_{-t-1} \omega\right)\right)\right\|^{2} \mathrm{~d} s+\int_{t}^{t+1} p_{2}\left(\theta_{\tau-t-1} \omega\right) \mathrm{d} \tau \\
& \quad+C \int_{t}^{t+1}\left(\left\|u\left(\tau, \theta_{-t-1} \omega, u_{0}\left(\theta_{-t-1} \omega\right)\right)\right\|^{2}+\left\|\nabla u\left(\tau, \theta_{-t-1} \omega, u_{0}\left(\theta_{-t-1} \omega\right)\right)\right\|^{2}\right. \\
& \left.\quad+\left\|u\left(\tau, \theta_{-t-1} \omega, u_{0}\left(\theta_{-t-1} \omega\right)\right)\right\|_{p}^{p}\right) \mathrm{d} \tau .
\end{aligned}
$$

By lemma 4.3 and 4.4, it follows from (4.37) and (4.35) that, for all $t \geq T_{B}(\omega)$ 


$$
\begin{aligned}
& \left\|\nabla v\left(t+1, \theta_{-t-1} \omega, v_{0}\left(\theta_{-t-1} \omega\right)\right)\right\|^{2} \leq C_{3}+C_{4} r(\omega)+\int_{-1}^{0} P_{2}\left(\theta_{s} \omega\right) \mathrm{d} s \\
& \leq C_{3}+C_{4} r(\omega)+\int_{-1}^{0}\left(C_{1} \mathrm{e}^{\frac{-\lambda}{2}} r(\omega)+c_{2}\right) \mathrm{d} s \leq C_{5}+C_{6} r(\omega) .
\end{aligned}
$$

Then by 4.38 and 3.9, we have, for all $t \geq T_{B}(\omega)$

$$
\begin{aligned}
& \left\|\nabla u\left(t+1, \theta_{-t-1} \omega, u_{0}\left(\theta_{-t-1} \omega\right)\right)\right\|^{2}=\left\|\nabla v\left(t+1, \theta_{-t-1} \omega, v_{0}\left(\theta_{-t-1} \omega\right)\right)+\nabla z(\omega)\right\|^{2} \\
& \leq 2\left\|\nabla v\left(t+1, \theta_{-t-1} \omega, v_{0}\left(\theta_{-t-1} \omega\right)\right)\right\|^{2}+2\|\nabla z(\omega)\|^{2} \leq C_{5}+C_{6} r(\omega),
\end{aligned}
$$

which completes the proof.

Lemma 4.6. Assume that $g^{j}, \quad g \in L^{2}\left(\mathbb{R}^{n}\right)$ and (3.3)-(3.5) hold, let $B=\{B(\omega)\}_{\omega \in \Omega} \in D$ and $u_{0}(\omega) \in B(\omega)$. Then for every $\epsilon>0$ and P-a.e $\omega \in \Omega$, there exists $T^{\star}=T_{B}^{\star}(\omega, \epsilon)>0$ and $\mathbb{R}^{\star}=\mathbb{R}^{\star}(\omega, \epsilon)>0$ such that the solution $v\left(t, \omega, v_{0}(\omega)\right)$ of (3.10) with $v_{0}(\omega)=u_{0}(\omega)-z(\omega)$ satisfies, for all $t \geq T^{\star}$

$$
\int_{|x| \geq \mathbb{R}^{\star}}\left|v\left(t, \theta_{-t} \omega, v_{0}\left(\theta_{-t} \omega\right)\right)(x)\right|^{2} \mathrm{~d} x \leq \epsilon .
$$

Proof. Choose a smooth function $\theta$ defined on $\mathbb{R}^{+}$such that $0 \leq \theta(s) \leq 1$ for all $s \in \mathbb{R}^{+}$and

$$
\theta(s)=\left\{\begin{array}{l}
0 \text { for } 0 \leq s \leq 1 \\
1 \text { for } s \geq 2
\end{array}\right.
$$

Then there exists a constant $C$ such that $\left|\theta^{\prime}(s)\right| \leq C$ for any $s \in \mathbb{R}^{+}$, multiplying (3.10) by $\theta\left(\frac{|x|^{2}}{k^{2}}\right) \cdot v$ in $L^{2}\left(\mathbb{R}^{n}\right)$, and integrating over $\mathbb{R}^{n}$ we find that

$$
\begin{aligned}
& \frac{1}{2} \frac{\mathrm{d}}{\mathrm{d} t} \int_{\mathbb{R}^{n}} \theta\left(\frac{|x|^{2}}{k^{2}}\right)|v|^{2} \mathrm{~d} x+\lambda \int_{\mathbb{R}^{n}} \theta\left(\frac{|x|^{2}}{k^{2}}\right)|v|^{2} \mathrm{~d} x-\int_{\mathbb{R}^{n}}(\Delta v) \theta\left(\frac{|x|^{2}}{k^{2}}\right) v \mathrm{~d} x \\
& =\int_{\mathbb{R}^{n}} \theta\left(\frac{|x|^{2}}{k^{2}}\right) f(u) v \mathrm{~d} x+\int_{\mathbb{R}^{n}}\left(g+\Delta z\left(\theta_{t} \omega\right)\right) \theta\left(\frac{|x|^{2}}{k^{2}}\right) v \mathrm{~d} x+\int_{\mathbb{R}^{n}} D_{j} g^{j} \theta\left(\frac{|x|^{2}}{k^{2}}\right) v \mathrm{~d} x .
\end{aligned}
$$

We now estimate the terms in (4.39) as follows, first we have

$$
\begin{aligned}
-\int_{\mathbb{R}^{n}}(\Delta v) \theta\left(\frac{|x|^{2}}{k^{2}}\right) v \mathrm{~d} x & =\int_{\mathbb{R}^{n}} \theta\left(\frac{|x|^{2}}{k^{2}}\right)|\nabla v|^{2} \mathrm{~d} x+\int_{\mathbb{R}^{n}} v \theta^{\prime}\left(\frac{|x|^{2}}{k^{2}}\right) \frac{2 x}{k^{2}} \cdot \nabla v \mathrm{~d} x \\
& =\int_{\mathbb{R}^{n}}|\nabla v|^{2} \theta\left(\frac{|x|^{2}}{k^{2}}\right) \mathrm{d} x+\int_{k \leq|x| \leq \sqrt{2 k}} v \theta^{\prime}\left(\frac{|x|^{2}}{k^{2}}\right) \frac{2 x}{k^{2}} \cdot \nabla v \mathrm{~d} x .
\end{aligned}
$$

Note that the second term on the right-hand side of (4.40) is bounded by

$$
\begin{aligned}
\left|\int_{k \leq \leq x \mid \leq \sqrt{2 k}} v \theta^{\prime}\left(\frac{|x|^{2}}{k^{2}}\right) \frac{2 x}{k^{2}} \cdot \nabla v \mathrm{~d} x\right| & \leq \frac{2 \sqrt{2}}{k} \int_{k \leq|x| \leq \sqrt{2 k}}|v| \theta^{\prime}\left(\frac{|x|^{2}}{k^{2}}\right)|\nabla v| \mathrm{d} x \\
& \leq \frac{C}{k} \int_{\mathbb{R}^{n}}|v||\nabla v| \mathrm{d} x \leq \frac{C}{k}\left(\|v\|^{2}+\|\nabla v\|^{2}\right) .
\end{aligned}
$$

By (4.40), (4.41), we find that

$$
-\int_{\mathbb{R}^{n}}(\Delta v) \theta\left(\frac{|x|^{2}}{k^{2}}\right) v \mathrm{~d} x \leq \int_{\mathbb{R}^{n}} \theta\left(\frac{|x|^{2}}{k^{2}}\right)|\nabla v|^{2} \mathrm{~d} x-\frac{C}{k}\left(\|v\|^{2}+\|\nabla v\|^{2}\right) .
$$


From (4.39) the first term on the right-hand side, we have

$$
\int_{\mathbb{R}^{n}} \theta\left(\frac{|x|^{2}}{k^{2}}\right) f(u) v \mathrm{~d} x=\int_{\mathbb{R}^{n}} \theta\left(\frac{|x|^{2}}{k^{2}}\right) f(u) u \mathrm{~d} x-\int_{\mathbb{R}^{n}} f(u) \theta\left(\frac{|x|^{2}}{k^{2}}\right) z\left(\theta_{t} \omega\right) \mathrm{d} x .
$$

By (3.3), the first term on the right-hand side of (4.43) is bounded by

$$
\int_{\mathbb{R}^{n}} \theta\left(\frac{|x|^{2}}{k^{2}}\right) f(u) u \mathrm{~d} x \leq-\alpha_{1} \int_{\mathbb{R}^{n}}|u|^{p} \theta\left(\frac{|x|^{2}}{k^{2}}\right) \mathrm{d} x+k_{1} \int_{\mathbb{R}^{n}}|u|^{2} \theta\left(\frac{|x|^{2}}{k^{2}}\right) \mathrm{d} x .
$$

By (3.4), the second term on the right-hand side of (4.43) is bounded by

$$
\begin{aligned}
& \left|\int_{\mathbb{R}^{n}} f(u) \theta\left(\frac{|x|^{2}}{k^{2}}\right) z\left(\theta_{t} \omega\right)\right| \mathrm{d} x \\
& \leq-\alpha_{3} \int_{\mathbb{R}^{n}}|u|^{p-1} \theta\left(\frac{|x|^{2}}{k^{2}}\right)\left|z\left(\theta_{t} \omega\right)\right| \mathrm{d} x+k_{3} \int_{\mathbb{R}^{n}}|u| \theta\left(\frac{|x|^{2}}{k^{2}}\right)\left|z\left(\theta_{t} \omega\right)\right| \mathrm{d} x \\
& \leq-\frac{1}{2} \alpha_{1} \int_{\mathbb{R}^{n}}|u|^{p} \theta\left(\frac{|x|^{2}}{k^{2}}\right) \mathrm{d} x+C \int_{\mathbb{R}^{n}}\left|z\left(\theta_{t} \omega\right)\right|^{p} \theta\left(\frac{|x|^{2}}{k^{2}}\right) \mathrm{d} x \\
& \quad+\frac{1}{2} k_{3} \int_{\mathbb{R}^{n}}\left|z\left(\theta_{t} \omega\right)\right|^{2} \theta\left(\frac{|x|^{2}}{k^{2}}\right) \mathrm{d} x+\frac{1}{2} k_{3} \int_{\mathbb{R}^{n}}|u|^{2} \theta\left(\frac{|x|^{2}}{k^{2}}\right) \mathrm{d} x .
\end{aligned}
$$

Then it follows from (4.43)-(4.45) we have that

$$
\begin{aligned}
& \int_{\mathbb{R}^{n}} f(u) \theta\left(\frac{|x|^{2}}{k^{2}}\right) v \mathrm{~d} x \leq-\frac{3}{2} \alpha_{1} \int_{\mathbb{R}^{n}}|u|^{p} \theta\left(\frac{|x|^{2}}{k^{2}}\right) \mathrm{d} x+k_{1} \int_{\mathbb{R}^{n}}|u|^{2} \theta\left(\frac{|x|^{2}}{k^{2}}\right) \mathrm{d} x \\
& +\frac{1}{2} k_{3} \int_{\mathbb{R}^{n}}|u|^{2} \theta\left(\frac{|x|^{2}}{k^{2}}\right) \mathrm{d} x+C \int_{\mathbb{R}^{n}}\left(\left|z\left(\theta_{t} \omega\right)\right|^{p}+\left|z\left(\theta_{t} \omega\right)\right|^{2}\right) \theta\left(\frac{|x|^{2}}{k^{2}}\right) \mathrm{d} x .
\end{aligned}
$$

For the second term on the right-hand side of (4.39) we have

$$
\begin{aligned}
& \left|\int_{\mathbb{R}^{n}}\left(g+\Delta z\left(\theta_{t} \omega\right)\right) \theta\left(\frac{|x|^{2}}{k^{2}}\right) v \mathrm{~d} x\right| \\
& \leq \frac{1}{2} \lambda \int_{\mathbb{R}^{n}} \theta\left(\frac{|x|^{2}}{k^{2}}\right)|v|^{2} \mathrm{~d} x+\frac{1}{\lambda} \int_{\mathbb{R}^{n}}\left(g^{2}+\left|\Delta z\left(\theta_{t} \omega\right)\right|^{2}\right) \theta\left(\frac{|x|^{2}}{k^{2}}\right) \mathrm{d} x .
\end{aligned}
$$

For the last term on the right-hand side of (4.39), we have that

$$
\begin{aligned}
\int_{\mathbb{R}^{n}} D_{j} g^{j} \theta\left(\frac{|x|^{2}}{k^{2}}\right) v \mathrm{~d} x & =-\int_{\mathbb{R}^{n}} \tilde{g} \frac{2 k}{k^{2}} \theta^{\prime}\left(\frac{|x|^{2}}{k^{2}}\right) v \mathrm{~d} x-\int_{\mathbb{R}^{n}} \tilde{g} \nabla v \theta\left(\frac{|x|^{2}}{k^{2}}\right) \mathrm{d} x \\
& \leq \frac{C}{k} \int_{\mathbb{R}^{n}}|\tilde{g}||v| \mathrm{d} x+\int_{\mathbb{R}^{n}}|\tilde{g}| \theta\left(\frac{|x|^{2}}{k^{2}}\right)|\nabla v| \mathrm{d} x \\
& \leq \frac{C}{k}\left(\|\tilde{g}\|^{2}+\|v\|^{2}\right)+\frac{1}{2} \lambda \int_{\mathbb{R}^{n}} \theta\left(\frac{|x|^{2}}{k^{2}}\right)|\tilde{g}|^{2} \mathrm{~d} x+\frac{1}{2 \lambda} \int_{\mathbb{R}^{n}} \theta\left(\frac{|x|^{2}}{k^{2}}\right)|\nabla v|^{2} \mathrm{~d} x .
\end{aligned}
$$

Finally, by (4.39), (4.42) and (4.47) (4.48), we have that 


$$
\begin{aligned}
& \frac{1}{2} \frac{\mathrm{d}}{\mathrm{d} t} \int_{\mathbb{R}^{n}} \theta\left(\frac{|x|^{2}}{k^{2}}\right)|v|^{2} \mathrm{~d} x+\frac{1}{2} \lambda \int_{\mathbb{R}^{n}} \theta\left(\frac{|x|^{2}}{k^{2}}\right)|v|^{2} \mathrm{~d} x \\
& +\frac{\lambda-1}{\lambda} \int_{\mathbb{R}^{n}} \theta\left(\frac{|x|^{2}}{k^{2}}\right)|\nabla v|^{2} \mathrm{~d} x-\frac{3}{2} \alpha_{1} \int_{\mathbb{R}^{n}}|u|^{p} \theta\left(\frac{|x|^{2}}{k^{2}}\right) \mathrm{d} x \\
& \leq \frac{C}{k}\left(\|g\|^{2}+2\|v\|^{2}+\mid \nabla v \|^{2}\right)+\int_{\mathbb{R}^{n}}\left(\left(\frac{2 k_{1}+k_{3}}{2}\right)|u|^{2}+\frac{1}{\lambda}|g|^{2}+\frac{1}{2} \lambda|\tilde{g}|^{2}\right) \theta\left(\frac{|x|^{2}}{k^{2}}\right) \mathrm{d} x \\
& \quad+\int_{\mathbb{R}^{n}}\left(C\left(\left|z\left(\theta_{t} \omega\right)\right|^{p}+\left|z\left(\theta_{t} \omega\right)\right|^{2}\right)+\frac{1}{\lambda}\left|\Delta z\left(\theta_{t} \omega\right)\right|^{2}\right) \theta\left(\frac{|x|^{2}}{k^{2}}\right) \mathrm{d} x .
\end{aligned}
$$

Note that (4.49) implies that

$$
\begin{aligned}
& \frac{\mathrm{d}}{\mathrm{d} t} \int_{\mathbb{R}^{n}} \theta\left(\frac{|x|^{2}}{k^{2}}\right)|v|^{2} \mathrm{~d} x+\lambda \int_{\mathbb{R}^{n}} \theta\left(\frac{|x|^{2}}{k^{2}}\right)|v|^{2} \mathrm{~d} x \\
& \leq \frac{C}{k}\left(\|g\|^{2}+2\|v\|^{2}+\|\nabla v\|^{2}\right)+\int_{\mathbb{R}^{n}}\left(\frac{2}{\lambda}|g|^{2}+2 \lambda|\tilde{g}|^{2}\right) \theta\left(\frac{|x|^{2}}{k^{2}}\right) \mathrm{d} x \\
& \quad+\int_{\mathbb{R}^{n}}\left(C\left(\left|z\left(\theta_{t} \omega\right)\right|^{p}+\left|z\left(\theta_{t} \omega\right)\right|^{2}\right)+\frac{2}{\lambda}\left|\Delta z\left(\theta_{t} \omega\right)\right|^{2}\right) \theta\left(\frac{|x|^{2}}{k^{2}}\right) \mathrm{d} x .
\end{aligned}
$$

By lemma 4.1 and 4.5, there is $T_{1}=T_{1}(B, \omega)>0$ such that for all $t \geq T_{1}$,

$$
\left\|v\left(t, \theta_{-t} \omega, v_{0}\left(\theta_{-t} \omega\right)\right)\right\|_{H^{1}\left(\mathbb{R}^{n}\right)}^{2} \leq C(1+r(\omega)) .
$$

Now integrating (4.50) over $\left(T_{1}, t\right)$ we get that, for all $t \geq T_{1}$

$$
\begin{aligned}
& \int_{\mathbb{R}^{n}} \theta\left(\frac{|x|^{2}}{k^{2}}\right)\left|v\left(t, \omega, v_{0}(\omega)\right)\right|^{2} \mathrm{~d} x \leq \mathrm{e}^{\lambda\left(T_{1}-t\right)} \int_{\mathbb{R}^{n}} \theta\left(\frac{|x|^{2}}{k^{2}}\right)\left|v\left(T_{1}, \omega, v_{0}(\omega)\right)\right|^{2} \mathrm{~d} x \\
& +\frac{C}{k} \int_{T_{1}}^{t} \mathrm{e}^{\lambda(s-t)}\left(\|g\|^{2}+\left.2\left\|\left.v\left(s, \omega, v_{0}(\omega)\right)\right|^{2}+\right\| \nabla v\left(s, \omega, v_{0}(\omega)\right)\right|^{2}\right) \mathrm{d} s \\
& +\int_{T_{1}}^{t} \mathrm{e}^{\lambda(s-t)} \int_{\mathbb{R}^{n}}\left(\frac{2}{\lambda}|g|^{2}+2 \lambda|\tilde{g}|^{2}\right) \theta\left(\frac{|x|^{2}}{k^{2}}\right) \mathrm{d} x \mathrm{~d} s \\
& +\int_{T_{1}}^{t} \mathrm{e}^{\lambda(s-t)} \int_{\mathbb{R}^{n}}\left(C\left(\left|z\left(\theta_{t} \omega\right)\right|^{p}+\left|z\left(\theta_{t} \omega\right)\right|^{2}\right)+\frac{2}{\lambda}\left|\Delta z\left(\theta_{t} \omega\right)\right|^{2}\right) \theta\left(\frac{|x|^{2}}{k^{2}}\right) \mathrm{d} x \mathrm{~d} s .
\end{aligned}
$$

Replacing $\omega$ by $\theta_{-t} \omega$, we obtain from (4.52) that, for all $t \geq T_{1}$,

$$
\begin{aligned}
& \int_{\mathbb{R}^{n}} \theta\left(\frac{|x|^{2}}{k^{2}}\right)\left|v\left(t, \theta_{-t} \omega, v_{0}\left(\theta_{-t} \omega\right)\right)\right|^{2} \mathrm{~d} x \leq \mathrm{e}^{\lambda\left(T_{1}-t\right)} \int_{\mathbb{R}^{n}} \theta\left(\frac{|x|^{2}}{k^{2}}\right)\left|v\left(T_{1}, \theta_{-t} \omega, v_{0}\left(\theta_{-t} \omega\right)\right)\right|^{2} \mathrm{~d} x \\
& +\frac{C}{k} \int_{T_{1}}^{t} \mathrm{e}^{\lambda(s-t)}\left(\|g\|^{2}+2\left|v\left(s, \theta_{-t} \omega, v_{0}\left(\theta_{-t} \omega\right)\right)\left\|^{2}+\right\| \nabla v\left(s, \theta_{-t} \omega, v_{0}\left(\theta_{-t} \omega\right)\right)\right|^{2}\right) \mathrm{d} s \\
& +\int_{T_{1}}^{t} \mathrm{e}^{\lambda(s-t)} \int_{\mathbb{R}^{n}}\left(\frac{2}{\lambda}|g|^{2}+2 \lambda|\tilde{g}|^{2}\right) \theta\left(\frac{|x|^{2}}{k^{2}}\right) \mathrm{d} x \mathrm{~d} s \\
& +\int_{T_{1}}^{t} \mathrm{e}^{\lambda(s-t)} \int_{\mathbb{R}^{n}}\left(\tilde{C}\left(\left|z\left(\theta_{s-t} \omega\right)\right|^{p}+\left|z\left(\theta_{s-t} \omega\right)\right|^{2}\right)+\frac{2}{\lambda}\left|\Delta z\left(\theta_{s-t} \omega\right)\right|^{2}\right) \theta\left(\frac{|x|^{2}}{k^{2}}\right) \mathrm{d} x \mathrm{~d} s .
\end{aligned}
$$


In what follows, we estimate the terms in (4.53). First replacing $t$ by $T_{1}$ and then replacing $\omega$ by $\theta_{-t} \omega$ in (4.10), we have the following bounds for the first term on the right-hand side of (4.53)

$$
\begin{aligned}
& \mathrm{e}^{\lambda\left(T_{1}-t\right)} \int_{\mathbb{R}^{n}} \theta\left(\frac{|x|^{2}}{k^{2}}\right)\left|v\left(T_{1}, \theta_{-t} \omega, v_{0}\left(\theta_{-t} \omega\right)\right)\right|^{2} \mathrm{~d} x \\
& \leq \mathrm{e}^{\lambda\left(T_{1}-t\right)}\left(\mathrm{e}^{-\lambda T_{1}}\left\|v_{0}\left(\theta_{-t} \omega\right)\right\|^{2}+\int_{0}^{T_{1}} \mathrm{e}^{\lambda\left(s-T_{1}\right)} P_{1}\left(\theta_{s-t} \omega\right) \mathrm{d} s+C\right) \\
& \leq \mathrm{e}^{-\lambda T_{1}}\left\|v_{0}\left(\theta_{-t} \omega\right)\right\|^{2}+C \mathrm{e}^{\lambda\left(T_{1}-t\right)}+\int_{-t}^{T_{1}-t} \mathrm{e}^{\lambda \tau} P_{1}\left(\theta_{\tau} \omega\right) \mathrm{d} \tau \\
& \leq \mathrm{e}^{-\lambda t}\left\|v_{0}\left(\theta_{-t} \omega\right)\right\|^{2}+C \mathrm{e}^{\lambda\left(T_{1}-t\right)}+\int_{-t}^{T_{1}-t} \mathrm{e}^{\frac{1}{2} \lambda \tau} C_{3} r(\omega) \mathrm{d} \tau \\
& \leq \mathrm{e}^{-\lambda t}\left\|v_{0}\left(\theta_{-t} \omega\right)\right\|^{2}+C \mathrm{e}^{\lambda\left(T_{1}-t\right)}+\frac{2}{\lambda} C_{3} r(\omega) \mathrm{e}^{\frac{1}{2} \lambda\left(T_{1}-t\right)},
\end{aligned}
$$

where we have used (4.7). By (4.54), we find that, given $\epsilon>0$, there is $T_{2}=(B, \omega, \epsilon)>T_{1}$ such that for all $t \geq T_{2}$

$$
\mathrm{e}^{\lambda\left(T_{1}-t\right)} \int_{\mathbb{R}^{n}} \theta\left(\frac{|x|^{2}}{k^{2}}\right)\left|v\left(T_{1}, \theta_{-t} \omega, v_{0}\left(\theta_{-t} \omega\right)\right)\right|^{2} \mathrm{~d} x \leq \epsilon .
$$

By lemma 4.2, there is $T_{3}=T_{3}(B, \epsilon)>T_{1}$ such that the fourth term on the right-hand side of (4.53) satisfies

$$
\frac{C}{k} \int_{T_{1}}^{t} \mathrm{e}^{\lambda(s-t)}\left\|\nabla v\left(s, \theta_{-t} \omega, v_{0}\left(\theta_{-t} \omega\right)\right)\right\|^{2} \mathrm{~d} s \leq \frac{C}{k}(1+r(\omega)) .
$$

And hence, there is $R_{1}=R_{1}(\omega, \epsilon)>0$ such that for all $t \geq T_{3}$ and $k \geq R_{1}$,

$$
\frac{C}{k} \int_{T_{1}}^{t} \mathrm{e}^{\lambda(s-t)}\left\|\nabla v\left(s, \theta_{-t} \omega, v_{0}\left(\theta_{-t} \omega\right)\right)\right\|^{2} \mathrm{~d} s \leq \epsilon .
$$

First replacing $t$ by $s$ and then replacing $\omega$ by $\theta_{-t} \omega$ in (4.10), we find that the third term on the right-hand side of (4.53) satisfies

$$
\begin{aligned}
& \frac{2 C}{k} \int_{T_{1}}^{t} \mathrm{e}^{\lambda(s-t)}\left\|v\left(s, \theta_{-t} \omega, v_{0}\left(\theta_{-t} \omega\right)\right)\right\|^{2} \mathrm{~d} s \\
& \leq \frac{2 C}{k} \int_{T_{1}}^{t} \mathrm{e}^{-\lambda t}\left\|v_{0}\left(\theta_{-t} \omega\right)\right\|^{2} \mathrm{~d} s+\frac{2 C}{k} \int_{T_{1}}^{t} \mathrm{e}^{\lambda(s-t)} \int_{0}^{s} \mathrm{e}^{\lambda(\tau-s)} P_{1}\left(\theta_{\tau-t} \omega\right) \mathrm{d} \tau \mathrm{d} s+\frac{2 C}{k} \int_{T_{1}}^{t} \mathrm{e}^{\lambda(s-t)} \mathrm{d} s \\
& \leq \frac{2 C}{k} \mathrm{e}^{-\lambda t}\left(t-T_{1}\right)\left\|v_{0}\left(\theta_{-t} \omega\right)\right\|^{2}+\frac{2 C}{k}+\frac{2 C}{k} \int_{T_{1}}^{t} \int_{0}^{s} \mathrm{e}^{\lambda(\tau-t)} P_{1}\left(\theta_{\tau} \omega\right) \mathrm{d} \tau \mathrm{d} s \\
& \leq \frac{2 C}{k} \mathrm{e}^{-\lambda t}\left(t-T_{1}\right)\left\|v_{0}\left(\theta_{-t} \omega\right)\right\|^{2}+\frac{2 C}{k}+\frac{2 C}{k} \int_{T_{1}}^{t} \int_{-t}^{s-t} \mathrm{e}^{\lambda \tau} P_{1}\left(\theta_{\tau} \omega\right) \mathrm{d} \tau \mathrm{d} s \\
& \leq \frac{2 C}{k} \mathrm{e}^{-\lambda t}\left(t-T_{1}\right)\left\|v_{0}\left(\theta_{-t} \omega\right)\right\|^{2}+\frac{2 C}{k}+\frac{2 C}{k} C_{3} r(\omega) \int_{T_{1}}^{t} \int_{-t}^{s-t} \mathrm{e}^{\frac{\lambda \tau}{2}} \mathrm{~d} \tau \mathrm{d} s \\
& \leq \frac{2 C}{k} \mathrm{e}^{-\lambda t}\left(t-T_{1}\right)\left\|v_{0}\left(\theta_{-t} \omega\right)\right\|^{2}+\frac{2 C}{k}+\frac{8 C}{\lambda^{2} k} C_{3} r(\omega) .
\end{aligned}
$$

This implies that there exist $T_{4}=T_{4}(B, \omega, \epsilon)>T_{1}$ and $R_{2}=R_{2}(\omega, \epsilon)$ such that for all $t \geq T_{4}$ and $k \geq R_{2}$,

$$
\frac{2 C}{k} \int_{T_{1}}^{t} \mathrm{e}^{\lambda(s-t)}\left\|v\left(s, \theta_{-t} \omega, v_{0}\left(\theta_{-t} \omega\right)\right)\right\|^{2} \mathrm{~d} s \leq \epsilon .
$$

Then the second term on the right-hand side of (4.53), there exist $T_{5}=T_{5}(B, \omega, \epsilon)>T_{1}$ and $R_{3}=R_{3}(\omega, \epsilon)$ such that for all $t \geq T_{5}$ and $k \geq R_{3}$ we have that

$$
\frac{C}{k} \int_{T_{1}}^{t} \mathrm{e}^{\lambda(s-t)}\|g\|^{2} \leq \epsilon, \frac{2 C \lambda}{k} \mathrm{e}^{\lambda\left(t-T_{1}\right)}\|g\|^{2} \leq \epsilon .
$$


Note that $g^{j}, g \in L^{2}\left(\mathbb{R}^{n}\right)$. therefore, there is $R_{4}=R_{4}(\epsilon)$ such that for all $k \geq R_{4}$,

$$
\int_{|x| \geq k}\left(\frac{2}{\lambda}|g|^{2}+2 \lambda|\tilde{g}|^{2}\right) \mathrm{d} x \leq \epsilon .
$$

For the five term on the right-hand side of (4.53), we have

$$
\begin{aligned}
& \int_{T_{1}}^{t} \mathrm{e}^{\lambda(s-t)} \int_{\mathbb{R}^{n}}\left(\frac{2}{\lambda}|g|^{2}+2 \lambda|\tilde{g}|^{2}\right) \theta\left(\frac{|x|^{2}}{k^{2}}\right) \mathrm{d} x \mathrm{~d} s \\
& \leq \int_{T_{1}}^{t} \mathrm{e}^{\lambda(s-t)} \int_{|x| \geq k}\left(\frac{2}{\lambda}|g|^{2}+2 \lambda|\tilde{g}|^{2}\right) \mathrm{d} x \mathrm{~d} s \leq \epsilon \\
& \int_{T_{1}}^{t} \mathrm{e}^{\lambda(s-t)} \mathrm{d} s \leq \epsilon .
\end{aligned}
$$

Note that $z\left(\theta_{t} \omega\right)=\sum_{j=1}^{m} h_{j} z_{j}\left(\theta_{t} \omega_{j}\right)$ and $h_{j} \in H^{2}\left(\mathbb{R}^{n}\right) \cap W^{2, p}\left(\mathbb{R}^{n}\right)$. Hence there is $R_{4}=R_{4}(\omega, \epsilon)$ such that for all $k \geq R_{4}$ and $j=1, \cdots, m$.

$$
\int_{|x| \geq k}\left(\left|h_{j}(x)\right|^{p}+\left|h_{j}(x)\right|^{2}+\left|\Delta h_{j}(x)\right|^{2}\right) \mathrm{d} x \leq \min \left\{\frac{\lambda \epsilon}{4 m p \tilde{C} r(\omega)}, \frac{\epsilon}{2 m^{2} r(\omega)}\right\},
$$

where $r(\omega)$ is the tempered function in (3.7) and $\tilde{C}$ is the positive constant in the last term on the right-hand side of (4.60), By (4.60) and (3.7), (3.8), we have the following bounds for the last term on the right-hand side of (4.53):

$$
\begin{aligned}
& \tilde{C} \int_{T_{1}}^{t} \mathrm{e}^{\lambda(s-t)} \int_{\mathbb{R}^{n}}\left(\left|z\left(\theta_{s-t} \omega\right)\right|^{2}+\left|z\left(\theta_{s-t} \omega\right)\right|^{p}+\left|\Delta z\left(\theta_{s-t} \omega\right)\right|^{2}\right) \theta\left(\frac{|x|^{2}}{k^{2}}\right) \mathrm{d} x \mathrm{~d} s \\
& \leq \tilde{C} \int_{T_{1}}^{t} \mathrm{e}^{\lambda(s-t)} \int_{|x| \geq k}\left(\left|z\left(\theta_{s-t} \omega\right)\right|^{2}+\left|z\left(\theta_{s-t} \omega\right)\right|^{p}+\left|\Delta z\left(\theta_{s-t} \omega\right)\right|^{2}\right) \mathrm{d} x \mathrm{~d} s \\
& \leq m p \tilde{C} \int_{T_{1}}^{t} \mathrm{e}^{\lambda(s-t)} \sum_{j=1}^{m} \int_{|x| \geq k}\left(\left|h_{j}\right|^{2}\left|z_{j}\left(\theta_{s-t}\right) \omega_{j}\right|^{2}+\left|h_{j}\right|^{p}\left|z_{j}\left(\theta_{s-t}\right) \omega_{j}\right|^{p}+\left|\Delta h_{j}\right|^{2}\left|z_{j}\left(\theta_{s-t}\right) \omega_{j}\right|^{2}\right) \mathrm{d} x \mathrm{~d} s \\
& \leq \frac{\lambda \epsilon}{2 r(\omega)} \int_{T_{1}}^{t} \mathrm{e}^{\lambda(s-t)} \sum_{j=1}^{m}\left(\left|z_{j}\left(\theta_{s-t}\right) \omega_{j}\right|^{2}+\left|z_{j}\left(\theta_{s-t}\right) \omega_{j}\right|^{p}\right) \mathrm{d} s \\
& \leq \frac{\lambda \epsilon}{2 r(\omega)} \int_{T_{1}}^{t} \mathrm{e}^{\lambda(s-t)} r\left(\theta_{s-t} \omega\right) \mathrm{d} s \leq \frac{\lambda \epsilon}{2 r(\omega)} \int_{T_{1}-t}^{0} \mathrm{e}^{\lambda \tau} r\left(\theta_{\tau} \omega\right) \mathrm{d} \tau \\
& \leq \frac{\lambda \epsilon}{2 r(\omega)} \int_{T_{1}-t}^{0} \mathrm{e}^{\frac{1}{2} \lambda \tau} r(\omega) \mathrm{d} \tau \leq \epsilon .
\end{aligned}
$$

Let $T_{5}=T_{5}(B, \omega, \epsilon)=\max \left\{T_{1}, \cdots, T_{5}\right\}$ and $R_{5}=R_{5}(\omega, \epsilon)=\max \left\{R_{1}, \cdots, R_{5}\right\}$. then it follows from (4.53), (4.55)-(4.61) that, for all $t \geq T_{5}$ and $k \geq R_{5}$, we have

$$
\int_{\mathbb{R}^{n}} \theta\left(\frac{|x|^{2}}{k^{2}}\right)\left|\nu\left(t, \theta_{-t} \omega, v_{0}\left(\theta_{-t} \omega\right)\right)\right|^{2} \mathrm{~d} x \leq 5 \epsilon,
$$

which shows that for all $t \geq T_{5}$ and $k \geq R_{5}$

$$
\int_{|x| \geq \sqrt{2 k}}\left|v\left(t, \theta_{-t} \omega, v_{0}\left(\theta_{-t} \omega\right)\right)\right|^{2} \mathrm{~d} x \leq \int_{\mathbb{R}^{n}} \theta\left(\frac{|x|^{2}}{k^{2}}\right)\left|v\left(t, \theta_{-t} \omega, v_{0}\left(\theta_{-t} \omega\right)\right)\right|^{2} \mathrm{~d} x \leq 5 \epsilon .
$$

This completes the proof.

Lemma 4.7. Assume that $g^{j}, g \in L^{2}\left(\mathbb{R}^{n}\right)$ and (3.3)-(3.5) hold. Let $B=\{B(\omega)\}_{\omega \in \Omega} \in D$ and $u_{0}(\omega) \in B(\omega)$. Then for every $\epsilon>0$ and P-a.e $\omega \in \Omega$, there exists $T^{\star}=T_{B}^{\star}(\omega, \epsilon)>0$ and $R^{\star}=R^{\star}(\omega, \epsilon)>0$ such that, for 
all $t \geq T^{\star}$

$$
\int_{|x| \geq R^{\star}}\left|u\left(t, \theta_{-t} \omega, u_{0}\left(\theta_{-t} \omega\right)\right)(x)\right|^{2} \mathrm{~d} x \leq \epsilon .
$$

Proof. Let $T^{\star}$ and $R^{\star}$ be the constant in lemma 4.6 By (4.60) and (3.7) we have, for all $t \geq T^{\star}$ and $k \geq R^{\star}$

$$
\begin{aligned}
\int_{|x| \geq R^{\star}}|z(\omega)|^{2} \mathrm{~d} x & =\int_{|x| \geq R^{\star}}\left|\sum_{j=1}^{m} h_{j} z_{j}\left(\omega_{j}\right)\right|^{2} \mathrm{~d} x \leq m^{2} \int_{|x| \geq R^{\star}}\left|h_{j}\right|^{2}\left|z_{j}\left(\omega_{j}\right)\right|^{2} \mathrm{~d} x \\
& \leq \frac{\epsilon}{2 r(\omega)} \sum_{j=1}^{m}\left|z_{j}\left(\omega_{j}\right)\right|^{2} \leq \frac{\epsilon}{2}
\end{aligned}
$$

then by (4.62) and lemma 4.6, we get that, for all $t \geq T^{\star}$ and $k \geq R^{\star}$

$$
\begin{aligned}
& \int_{|x| \geq R^{\star}}\left|u\left(t, \theta_{-t} \omega, u_{0}\left(\theta_{-t} \omega\right)\right)\right|^{2} \mathrm{~d} x=\int_{|x| \geq R^{\star}}\left|v\left(t, \theta_{-t} \omega, v_{0}\left(\theta_{-t} \omega\right)\right)+z(\omega)\right|^{2} \mathrm{~d} x \\
& =2 \int_{|x| \geq R^{\star}}\left|v\left(t, \theta_{-t} \omega, v_{0}\left(\theta_{-t} \omega\right)\right)\right|^{2} \mathrm{~d} x+2 \int_{|x| \geq R^{\star}}|z(\omega)|^{2} \mathrm{~d} x \leq 3 \epsilon,
\end{aligned}
$$

which completes the proof.

\section{Random Attractors}

In this section, we prove the existence of a $D$-random attractor for the random dynamical system $\phi$ associated with the stochastic reaction-diffusion Equations (3.1), (3.2) on $\mathbb{R}^{n}$. It follows from lemma 4.1 that $\phi$ has a closed random absorbing set in $D$, which along with the $D$-pullback asymptotic compactness will imply the existence of a unique $D$-random attractor. The $D$-pullback asymptotic compactness of $\phi$ is given below and will be proved by using the uniform estimates on the tails of solutions.

Lemma 5.1. Assume that $g^{j}, g \in L^{2}\left(\mathbb{R}^{n}\right)$ and (3.3)-(3.5) hold. Then the random dynamical system $\phi$ is $D$ pullback asymptotically compact in $L^{2}\left(\mathbb{R}^{n}\right)$; that is, for P-a.e $\omega \in \Omega$, the sequence $\left\{\phi\left(t_{n}, \theta_{-t_{n}} \omega, u_{0, n}\left(\theta_{-t_{n}} \omega\right)\right)\right\}$ has a convergent subsequence in $L^{2}\left(\mathbb{R}^{n}\right)$ provided $t_{n} \rightarrow \infty, B=\{B(\omega)\}_{\omega \in \Omega} \in D$ and $u_{0, n}\left(\theta_{-t_{n}} \omega\right) \in B\left(\theta_{-t_{n}} \omega\right)$.

Proof. Let $t_{n} \rightarrow \infty \quad B=\{B(\omega)\}_{\omega \in \Omega} \in D$ and $u_{0, n}\left(\theta_{-t_{n}} \omega\right) \in B\left(\theta_{-t_{n}} \omega\right)$. Then by lemma 4.1 for P-a.e $\omega \in \Omega$, we have that

$$
\left\{\phi\left(t_{n}, \theta_{-t_{n}} \omega, u_{0, n}\left(\theta_{-t_{n}} \omega\right)\right)\right\}_{n=1}^{\infty} \text { is bounded in } L^{2}\left(\mathbb{R}^{n}\right) .
$$

Hence, there is $\eta \in L^{2}\left(\mathbb{R}^{n}\right)$ such that, up to a subsequence,

$$
\phi\left(t_{n}, \theta_{-t_{n}} \omega, u_{0, n}\left(\theta_{-t_{n}} \omega\right)\right) \rightarrow \eta \text { weakly in } L^{2}\left(\mathbb{R}^{n}\right) .
$$

Next, we prove the weak convergence of (5.1) is actually strong convergence. Given $\epsilon>0$, by lemma 4.7, there is $T_{1}=T_{1}(B, \omega, \epsilon)$ and $R_{1}=R_{1}(\omega, \epsilon)$ such that for all $t \geq T_{1}$,

$$
\int_{|x| \geq R_{1}}\left|\phi\left(t, \theta_{-t} \omega, u_{0}\left(\theta_{-t} \omega\right)\right)\right|^{2} \mathrm{~d} x \leq \epsilon .
$$

Since $t_{n} \rightarrow \infty$, there is $N_{1}=N_{1}(B, \omega, \epsilon)$ such that $t_{n} \geq T_{1}$ for every $n \geq N_{1}$. Hence, it follows from (5.2) that for all $n \geq N_{1}$,

$$
\int_{|x| \geq R_{1}}\left|\phi\left(t_{n}, \theta_{-t_{n}} \omega, u_{0, n}\left(\theta_{-t_{n}} \omega\right)\right)\right|^{2} \mathrm{~d} x \leq \epsilon .
$$

On the other hand, by lemma 4.1 and 4.5, there $T_{2}=T_{2}(B, \omega)$ such that for all $t \geq T_{2}$,

$$
\left\|\phi\left(t, \theta_{-t} \omega, u_{0}\left(\theta_{-t} \omega\right)\right)\right\|_{H^{1} \mathbb{R}^{n}}^{2} \leq C(1+r(\omega)) .
$$

Let $N_{2}=N_{2}(\omega, \epsilon)$ be large enough such that $t_{n} \geq T_{2}$ for $n \geq N_{2}$. then by (5.4) we have that, for all $n \geq N_{2}$, 


$$
\left\|\phi\left(t_{n}, \theta_{-t_{n}} \omega, u_{0, n}\left(\theta_{-t_{n}} \omega\right)\right)\right\|_{H^{1}\left(\mathbb{R}^{n}\right)}^{2} \leq C(1+r(\omega)) .
$$

Denote by $Q_{R_{1}}$ the set $\left\{x \in \mathbb{R}^{n}:|x| \leq R_{1}\right\}$. By the compactness of embedding $H^{1}\left(Q_{R_{1}}\right) \rightarrow L^{2}\left(Q_{R_{1}}\right)$, it follows from (5.5) that, up to a subsequence,

$$
\phi\left(t_{n}, \theta_{-t_{n}} \omega, u_{0, n}\left(\theta_{-t_{n}} \omega\right)\right) \rightarrow \eta \quad \text { strogly in } L^{2}\left(Q_{R_{1}}\right),
$$

which shows that for the given $\epsilon>0$, there exists $N_{3}=N_{3}(B, \omega, \epsilon)$ such that for all $n \geq N_{3}$,

$$
\left\|\phi\left(t_{n}, \theta_{-t_{n}} \omega, u_{0, n}\left(\theta_{-t_{n}} \omega\right)\right)-\eta\right\|_{L^{2}\left(Q_{R_{1}}\right)}^{2} \leq \epsilon .
$$

Note that $\eta \in L^{2}\left(\mathbb{R}^{n}\right)$. Therefore there exists $R_{2}=R_{2}(\epsilon)$ such that

$$
\int_{|x| \geq R_{2}}|\eta(x)|^{2} \mathrm{~d} x \leq \epsilon .
$$

let $R_{3}=\max \left\{R_{1}, R_{2}\right\}$ and $N_{4}=\max \left\{N_{1}, N_{2}, N_{3}\right\}$. By (5.3), (5.6), and (6.7), we find that for all $n \geq N_{4}$,

$$
\begin{aligned}
& \left\|\phi\left(t_{n}, \theta_{-t_{n}} \omega, u_{0, n}\left(\theta_{-t_{n}} \omega\right)\right)-\eta\right\|_{L^{2}\left(Q_{R_{1}}\right)}^{2} \\
& \leq \int_{|x| \geq R_{3}}\left|\phi\left(t_{n}, \theta_{-t_{n}} \omega, u_{0, n}\left(\theta_{-t_{n}} \omega\right)\right)-\eta\right|^{2} \mathrm{~d} x+\int_{|x| \geq R_{3}}\left|\phi\left(t_{n}, \theta_{-t_{n}} \omega, u_{0, n}\left(\theta_{-t_{n}} \omega\right)\right)-\eta\right|^{2} \mathrm{~d} x \leq 5 \epsilon,
\end{aligned}
$$

which shows that

$$
\phi\left(t_{n}, \theta_{-t_{n}} \omega, u_{0, n}\left(\theta_{-t_{n}} \omega\right)\right) \rightarrow \eta \text { strong in } L^{2}\left(\mathbb{R}^{n}\right),
$$

as wanted.

Now we are in a position to present our main result: the existence of a $D$-random attractor for $\phi$ in $L^{2}\left(\mathbb{R}^{n}\right)$.

Theorem 5.2. Assume that $g^{j}, g \in L^{2}\left(\mathbb{R}^{n}\right)$ and (3.3)-(3.5) hold. Then the random dynamical system $\phi$ has a unique $D$-random attractor in $\mathbb{R}^{n}$.

Proof. Notice that $\phi$ has a closed random absorbing set $\{K(\omega)\}_{\omega \in \Omega}$ in $D$ by lemma 4.1, and is $D$-pullback asymptotically compact in $\mathbb{R}^{n}$ by lemma 5.1. Hence the existence of a unique $D$-random attractor for $\phi$ follows from proposition 2.7 immediately.

\section{Foundation Term}

This work was supported by the NSFC (11101334).

\section{References}

[1] Flandoli, F. and Schmalfuß, B. (1996) Random Attractors for the 3D Stochastic Navier-Stokes Equation with Multiplicative Noise. Stochastics and Stochastic Reports, 59, 21-45. http://dx.doi.org/10.1080/17442509608834083

[2] Antoci, F. and Prizzi, M. (2001) Reaction-Diffusion Equations on Unbounded Thin Domains. Topological Methods in Nonlinear Analysis, 18, 283-302.

[3] Hale, J.K. (1988) Asymptotic Behavior of Dissipative Systems. American Mathematical Society, Providence.

[4] Robinson, J.C. (2001) Infinite-Dimensional Dynamical Systems. Cambridge University Press, Cambridge. http://dx.doi.org/10.1007/978-94-010-0732-0

[5] Wang, B. (1999) Attractors for Reaction-Diffusion Equations in Unbounded Domains. Physica D, 128, 41-52. http://dx.doi.org/10.1016/S0167-2789(98)00304-2

[6] Crauel, H., Debussche, A. and Flandoli, F. (1997) Random Attractors. Journal of Dynamics and Differential Equations, 9, 307-341. http://dx.doi.org/10.1007/BF02219225

[7] Caraballo, T., Langa, J.A. and Robinson, J.C. (2001) A Stochastic Pitchfork Bifurcation in a Reaction-Diffusion Equation. Proceedings of the Royal Society A, 457, 2041-2061. http://dx.doi.org/10.1098/rspa.2001.0819 
[8] Bates, P.W., Lu, K.N. and Wang, B.X. (2009) Random Attractors for Stochastic Reaction-Diffusion Equations on Unbounded Domains. Journal of Differential Equations, 246, 845-869. http://dx.doi.org/10.1016/j.jde.2008.05.017

[9] Rosa, R. (1998) The Global Attractor for the 2D Navier-Stokes Flow on Some Unbounded Domains. Nonlinear Analysis, 32, 71-85. http://dx.doi.org/10.1016/S0362-546X(97)00453-7

[10] Ball, J.M. (2004) Global Attractors for Damped Semilinear Wave Equations. Discrete and Continuous Dynamical Systems, 10, 31-52. http://dx.doi.org/10.3934/dcds.2004.10.31

[11] Arnold, L. (1998) Random Dynamical Systems. Springer-Verlag, Berlin. http://dx.doi.org/10.1007/978-3-662-12878-7

[12] Crauel, H., Debussche, A. and Flandoli, F. (1997) Random Attractors. Journal of Dynamics and Differential Equations 9, 307-341. http://dx.doi.org/10.1007/BF02219225

[13] Crauel, H. and Flandoli, F. (1994) Attractors for Random Dynamical Systems. Probability Theory and Related Fields, 100, 365-393. http://dx.doi.org/10.1007/BF01193705 